

\title{
Beyond Shallow Water: appraisal of a numerical approach to hydraulic jumps based upon the Boundary Layer Theory
}

Francesco de Vita, Pierre-Yves Lagrée, Sergio Chibbaro, Stéphane Popinet

\section{To cite this version:}

Francesco de Vita, Pierre-Yves Lagrée, Sergio Chibbaro, Stéphane Popinet. Beyond Shallow Water: appraisal of a numerical approach to hydraulic jumps based upon the Boundary Layer Theory. European Journal of Mechanics - B/Fluids, 2019, 79, pp.233-246. 10.1016/j.euromechflu.2019.09.010 . hal-02295398

\section{HAL Id: hal-02295398 \\ https://hal.science/hal-02295398}

Submitted on 24 Sep 2019

HAL is a multi-disciplinary open access archive for the deposit and dissemination of scientific research documents, whether they are published or not. The documents may come from teaching and research institutions in France or abroad, or from public or private research centers.
L'archive ouverte pluridisciplinaire HAL, est destinée au dépôt et à la diffusion de documents scientifiques de niveau recherche, publiés ou non, émanant des établissements d'enseignement et de recherche français ou étrangers, des laboratoires publics ou privés. 


\title{
Beyond Shallow Water: appraisal of a numerical approach to hydraulic jumps based upon the Boundary Layer Theory.
}

\author{
Francesco De Vita ${ }^{\mathrm{a}, *}$, Pierre-Yves Lagrée ${ }^{\mathrm{b}}$, Sergio Chibbaro ${ }^{\mathrm{b}}$, Stéphane Popinet $^{\mathrm{b}}$ \\ ${ }^{a}$ Linné FLOW Centre and SeRC (Swedish e-Science Research Centre), KTH Mechanics, S-100 44 Stockholm, Sweden \\ ${ }^{b}$ Sorbonne Université, CNRS, Institut Jean le Rond d'Alembert, 75005 Paris France
}

\begin{abstract}
We study the flow of a thin layer of fluid over a flat surface. Commonly, the 1-D Shallow-water or SaintVenant set of equations are used to compute the solution of such flows. These simplified equations may be obtained through the integration of the Navier-Stokes equations over the depth of the fluid, but their solution requires the introduction of constitutive relations based on strict hypothesis on the flow régime. Here, we present an approach based on a kind of boundary layer system with hydrostatic pressure. This relaxes the need for closure relations which are instead obtained as solutions of the computation. It is then demonstrated that the corresponding closures are very dependent on the type of flow considered, for example laminar viscous slumps or hydraulic jumps. This has important practical consequences as far as the applicability of standard closures is concerned.
\end{abstract}

Keywords: Shallow Water, Saint-Venant, boundary layer flows.

\section{Introduction}

The "shallow water equations" or "Saint-Venant Equations", from the author of the first proposition [1, are a classical model useful for a large variety of practical configurations in coastal and hydraulic engineering. For example, they are used to predict flows in rivers, in open channels, in lakes, in shallow seas. Floods are simulated with the shallow water equations, as well as tides and many other environmental applications (see for instance Chanson's book [2]). The depth averaging strategy to obtain them is also used for many nonNewtonian flows [3] useful in industrial (concrete) or environmental applications (mud flows, avalanches). Moreover, the Saint-Venant equations are an hyperbolic system analogous to compressible gas flow so that the problem has some universality [4].

Nevertheless, the Saint-Venant equations are based on vertical averaging, which gives rise to several problems as it over-simplifies the physics. One of the approximation comes from the hypothesis of small depth compared to the length of the phenomena. This fundamental hypothesis is not relaxed here, but it is known that if depth increases, dispersive effects appear (the celerity of the waves depends on their wavelength [5]). What will be discussed here is the fact that one needs strong hypothesis on the shape of the velocity profile and on the wall shear stress to close the system of equations. Indeed, the Saint-Venant equations were originally proposed on a phenomenological basis. In [6] an asymptotic analysis is proposed to derive them from the 2D Navier-Stokes equations with mixed boundary conditions. In that derivation, only the laminar case is considered and the derived one-dimensional unclosed equations are closed primarily through a simple constant velocity assumption. Since then, while some attempts have been made to justify the different approximations and to point out more general non-constant closures [7, most often the constant closure is retained in practical computations [8. All the numerical schemes set the so called Boussinesq

\footnotetext{
* Corresponding author

Email address: fdv@mech.kth.se (Francesco De Vita)
} 
coefficient (which accounts for the non-uniform velocity profile in the transverse direction) to one; recently, 9 proposed to artificially increase the Boussinesq coefficient in order to reduce oscillations in transcritical flows or unsteady flows over frictional beds. The influence of the modelling of the wall shear stress has been recently discussed for jumps in water and granular flows, where closure is very different. [10].

Furthermore, the range of application of the Saint-Venant model is notably limited because it does not describe the vertical profile of the horizontal velocity. For this reason, the multilayer approach to the Shallow Water equations has been developed, and in particular in the form of numerical schemes for a set of SaintVenant-like systems. It consist in dividing the liquid depth in layers, each one described by its own height 30 and velocity [11, 12, thus modelling the fluid as composed of layers of immiscible liquids. Mass exchanges between layers have also been considered 13, 14, 15. From a numerical point of view, the global stability of weak solutions for the method proposed in [13 has been demonstrated in 16, while new efficient techniques have been recently developed [17, 18, 19. Concerning applications beyond Newtonian fluids, a multilayer method with $\mu(I)$ rheology and side walls friction has also been derived [20]. It is important to note that since these multilayer schemes have been developed as mathematical / numerical schemes, less attention has been paid to the physical boundary conditions and the relevant friction coefficients. An alternative method is also worth mentioning, consisting in performing a gradient expansion of the depth-averaged Navier-Stokes equations which gives a system of equations for the depth, the flow rate and an additional variable which accounts for the deviation of the wall shear from the shear corresponding to a parabolic velocity profile 40 21, 22]. This approach has had some success in particular in the analysis of the motion of viscous liquid films.

Here we follow a different path to present an unified picture of the problem. We hope in this way to shed some light on the links between different approaches, which are either more physically or mathematically grounded. Starting from the Saint-Venant model, we would like to address the issue of the value of the Boussinesq coefficient. In order to get information on this coefficient, we deal with a reduced system obtained from the Navier-Stokes equations using an asymptotic thin-layer expansion, which results in fact in the classical boundary layer or Prandtl equations. From a conceptual point of view, it means that the domain may be divided into two physically separated regions: an ideal fluid and a viscous boundary layer [23. The equations we obtain asymptotically, are actually the same already proposed to tackle the problem of the standing hydraulic jump on phenomenological grounds 24, when considering the limit of infinite Reynolds number. In particular, Higuera [25, 26] was the first to use these boundary layer equations to study a viscous hydraulic jump. This is an important test case that we shall repeat in the present work with a different numerical approach.

This simplified set of boundary layer or Prandtl equations will allow to compute directly the shape factor and the wall shear stress, whereas they are parameters in standard Saint-Venant approaches. Indeed, from a practical point of view, one success of the Shallow Water equations is its ability to describe a standing jump. This is known as Bélanger equations [27. Indeed, as shown by Watson [28, the position of the standing jump within the Saint-Venant description depends on the modelling of viscous effects. Many authors have tried, since then, to understand the structure of the radially-symmetric or 2D hydraulic jump [29, 30, 31] using various techniques issued from simplified boundary layer theory [32, 29, 33. At the same time, thanks to 2D Navier-Stokes computations, Dasgupta et al. 34. were able to simulate completely the problem, while a similar analysis was performed for a flow over a bump [35. In this work, we will characterise clearly what is the actual friction in terms of the Boussinesq coefficients in order to assess the validity of the different hypothesis usually adopted together with the Saint-Venant model. Besides, similar closure problems are encountered in different physical phenomena: granular flows when modelled by a Savage-Hutter depthaveraged model [36; or flows in arteries when modelled by averaging over the circular cross section. Note that the same idea has already been applied for these problems [37, 38, 39].

Starting from a physically-sound model, we need a numerical scheme to discretize and simulate it. It turns out that the natural scheme for our model is the one proposed for the multi-layer Saint-Venant equations 13, 14 with the introduction of an appropriate boundary condition that allows to compute the wall shear stress without the input of friction coefficients. This is fundamental for the proper description of transcritical flows or hydraulic jumps where errors in the quantification of the bottom friction and in the reconstruction of the vertical velocity profile can lead to instabilities or overestimation of the dissipation. 
The aim of this paper is thus to present in a general way a theoretical boundary layer model coupled with a versatile numerical model [13, 14. The relation between the boundary layer model and the numerical multi-layer schemes developed for the Saint-Venant equations will therefore be emphasized. The ensemble constitutes a general framework applicable to a wide range of phenomena. For instance, a boundary layer interacting with an external flow may lead to a "jump" in several different contexts 40: it was first observed by [41] for compressible flows, and has been identified by [42, 43, 44, for boundary layer mixed convection flows. This behaviour often corresponds to a "triple deck" structure [45, 46]. To support this view, we will recompute the Higuera solution, and we will present several other similar test cases while comparing the numerical solutions with the analytical ones whenever possible. We also discuss in some details the numerical scheme used to solve the equations via the free solver Basilisk [47.

The paper is organised as follows: in the first section, the Navier-Stokes equations are presented with their thin layer approximation leading to the Reduced Navier-Stokes set, which are Prandtl equations with specific boundary conditions and hydrostatic pressure. This system is integrated over the depth to obtain the Saint-Venant equations. Then, in the second section, the numerical "multilayer technique" is presented to solve the system. In the third section, some viscous slump flows are presented (Huppert slumps), then the Higuera standing jump solution is re-simulated. Finally, the influence of a bottom slope on the position of the standing jump is presented.

\section{Governing equations}

\subsection{Navier-Stokes equations}

The overall multiphase flow problem of two fluids (say a liquid and a gas) with a separating free surface over a given bottom may be simplified if one of the fluid is much heavier than the other. In this case, free surface flow phenomena can be fully described by the incompressible Navier-Stokes equations for the heavy fluid only, with proper boundary conditions at the interface. For simplicity we will consider a twodimensional flow with $x$ the horizontal axis and $z$ the vertical axis, pointing upward. The location of the free surface is denoted as $\eta(x, t)$, and the position of the bottom (or bathymetry) is denoted as $z_{b}(x)$, so that the depth is $h=\eta-z_{b}$. Across the depth the Navier-Stokes equations can be written as:

$$
\frac{\partial \mathbf{u}}{\partial t}+\mathbf{u} \cdot \nabla \mathbf{u}=-\frac{1}{\rho} \nabla p+\nu_{0} \nabla^{2} \mathbf{u}+\mathbf{f}, \quad \nabla \cdot \mathbf{u}=0
$$

where $\mathbf{u}=(u, w)$ is the velocity field, $p$ the pressure, $\nu_{0}$ the kinematic viscosity, $\rho$ the mass density $\left(\mu=\rho \nu_{0}\right.$ is the dynamic viscosity), and $\mathbf{f}=-g \hat{\mathbf{z}}$ the gravitational force. The two boundary conditions closing the system of equations (1) are the kinematic boundary condition at the free surface

$$
\frac{\partial \eta}{\partial t}+u_{s} \frac{\partial \eta}{\partial x}-w_{s}=0
$$

with no tangential stress at the surface and continuity of the normal stress, and the impermeability condition at the bottom (and no slip for viscous flow)

$$
u_{b} \frac{\partial z_{b}}{\partial x}-w_{b}=0
$$

The subscripts $s$ and $b$ denote quantities at the free surface and at the bottom respectively. Let us rescale the equations (1) introducing two characteristic dimensions $h_{0}$ (typical depth) and $L$ (a typical evolution length), in the $z$ and $x$ direction respectively, a typical wave amplitude $a_{s}$ and a characteristic wave speed $c_{0}=\sqrt{g h_{0}}$. With these quantities we can define two dimensionless parameters:

$$
\varepsilon=\frac{h_{0}}{L} \quad \text { and } \quad \delta=\frac{a_{s}}{h_{0}}
$$

we do not take into account the possibility of dispersion leading to solitary waves [5, 48]. The classical Saint-Venant derivation assumes the characteristic length in the vertical direction $z$ to be smaller than in 
the horizontal direction, $i$. e. $\varepsilon \ll 1$, and $\delta=\mathcal{O}(1)$ which allows to produce jumps. On the contrary, the Airy linearised wave theory on arbitrary depth requires $\varepsilon=\mathcal{O}(1)$ and $\delta \ll 1$. With the scales defined above it is possible to make dimensionless all the quantities in the Navier-Stokes equations:

$$
\tilde{x}=\frac{x}{L}=\frac{\varepsilon x}{h_{0}}, \quad \tilde{z}=\frac{z}{h_{0}}, \quad \tilde{t}=\frac{\varepsilon c_{0} t}{h_{0}}, \quad \tilde{u}=\frac{u}{c_{0}} \quad \text { and } \quad \tilde{w}=\frac{w}{\varepsilon c_{0}}
$$

where scales of time and transverse velocity are chosen assuming that inertial terms are dominant over viscous ones. For pressure, assuming the reference pressure to be zero at the surface, the following scales are taken:

$$
\tilde{p}=\frac{p}{\rho g h_{0}}, \quad \tilde{\eta}=\frac{\eta}{h_{0}}, \quad \tilde{h}=\frac{h}{h_{0}},
$$

Thus the rescaled system of Navier-Stokes equations is:

$$
\begin{aligned}
& \frac{\partial \tilde{u}}{\partial \tilde{x}}+\frac{\partial \tilde{w}}{\partial \tilde{z}}=0 \\
& \frac{\partial \tilde{u}}{\partial \tilde{t}}+\frac{\partial \tilde{u}^{2}}{\partial \tilde{x}}+\frac{\partial \tilde{u} \tilde{w}}{\partial \tilde{z}}=-\frac{\partial \tilde{p}}{\partial \tilde{x}}+\frac{\mu}{\varepsilon \rho c_{0} h_{0}}\left(\frac{\partial^{2} \tilde{u}}{\partial \tilde{z}^{2}}+\varepsilon^{2} \frac{\partial^{2} \tilde{u}}{\partial \tilde{x}^{2}}\right) \\
& \varepsilon^{2}\left(\frac{\partial \tilde{w}}{\partial \tilde{t}}+\frac{\partial \tilde{u} \tilde{w}}{\partial \tilde{x}}+\frac{\partial \tilde{w}^{2}}{\partial \tilde{z}}\right)=-\frac{\partial \tilde{p}}{\partial \tilde{z}}-1+\varepsilon \frac{\mu}{\rho c_{0} h_{0}}\left(\frac{\partial^{2} \tilde{w}}{\partial \tilde{z}^{2}}+\varepsilon^{2} \frac{\partial^{2} \tilde{w}}{\partial \tilde{x}^{2}}\right)
\end{aligned}
$$

Note that the topography variations are supposed compatible with the long-wave hypothesis: $\frac{\partial z_{b}}{\partial x}=\varepsilon \frac{\partial \tilde{z}_{b}}{\partial \tilde{x}}$. Note as well that the Froude number is one by construction, since we are considering flows with a single velocity scale. The velocity $\tilde{u}$ can still be smaller or larger than one, as a result of the computation.

\subsection{Reduced Navier-Stokes equations in the boundary-layer approximation}

Since we have assumed that $\varepsilon<<1$, equations (4) can be simplified through elimination of the terms of order $\mathcal{O}(\varepsilon)$ and, defining Reynolds number $R e=\varepsilon \rho c_{0} h_{0} / \mu$ which may be large or small (but not smaller than $\varepsilon)$, gives:

$$
\begin{aligned}
\frac{\partial \tilde{u}}{\partial \tilde{x}}+\frac{\partial \tilde{w}}{\partial \tilde{z}} & =0 \\
\frac{\partial \tilde{u}}{\partial \tilde{t}}+\frac{\partial \tilde{u}^{2}}{\partial \tilde{x}}+\frac{\partial \tilde{u} \tilde{w}}{\partial \tilde{z}} & =-\frac{\partial p}{\partial \tilde{x}}+\frac{1}{R e} \frac{\partial^{2} \tilde{u}}{\partial \tilde{z}^{2}} \\
0 & =-\frac{\partial p}{\partial \tilde{z}}-1
\end{aligned}
$$

This system of equation has the following boundary conditions at the free surface $\tilde{z}=\tilde{z}_{b}+\tilde{h}(\tilde{x}, \tilde{t})$, namely velocity of interface, reference pressure, and no shear stress:

$$
\tilde{w}=\frac{\partial \tilde{\eta}}{\partial t}+\tilde{u} \frac{\partial \tilde{\eta}}{\partial \tilde{x}}, \quad \tilde{p}\left(\tilde{x}, \tilde{z}=\tilde{z}_{b}+\tilde{h}(\tilde{x}, \tilde{t})\right)=0, \quad \frac{\partial \tilde{u}}{\partial \tilde{z}}=0,
$$

and at the solid bottom $\tilde{z}=\tilde{z}_{b}$ there is the no-slip boundary condition for both $\tilde{u}=0$ and $\tilde{w}=0$. The set of equations (5a $5 \mathrm{c}$ ) are the Prandtl equations for boundary-layer flows, and for this reason we call them Reduced Navier-Stokes Prandtl equations (RNSP). Together with the above boundary conditions, they are the system which we employ in this study. 


\subsection{RNSP equations with Prandtl transposition theorem}

Note that the classical Prandtl transposition theorem may be used here 49 ; it consists in changing $\tilde{z}$ in $\tilde{z}-\tilde{z}_{b}$, while $\tilde{u}$ is unchanged, and $\tilde{w}$ is replaced by $\tilde{w}-\frac{\partial \tilde{z}_{b}}{\partial \tilde{x}} \tilde{u}$. With this transformation, the no-slip boundary condition is at $\tilde{z}=0$. The pressure term $-\frac{\partial \tilde{p}}{\partial \tilde{x}}$ changes to (using the chain rule derivative and $5 \mathrm{c}$ ):

$$
-\left(\frac{\partial \tilde{p}}{\partial \tilde{x}}-\frac{\partial \tilde{z}_{b}}{\partial \tilde{x}} \frac{\partial \tilde{p}}{\partial \tilde{y}}\right)=-\frac{\partial \tilde{p}}{\partial \tilde{x}}-\frac{\partial \tilde{z}_{b}}{\partial \tilde{x}}
$$

Hence the momentum equation reads:

$$
\frac{\partial \tilde{u}}{\partial \tilde{t}}+\frac{\partial \tilde{u}^{2}}{\partial \tilde{x}}+\frac{\partial \tilde{u} \tilde{w}}{\partial \tilde{z}}=-\frac{\partial \tilde{p}}{\partial \tilde{x}}-\frac{\partial \tilde{z}_{b}}{\partial \tilde{x}}+\frac{1}{R e} \frac{\partial^{2} \tilde{u}}{\partial \tilde{z}^{2}},
$$

where $\tilde{z}=0$ is now the bottom and $\tilde{z}=\tilde{h}$ the free surface.

\subsection{Shallow Water or Saint-Venant equations}

The set of equations 5 (5a)- $5 \mathrm{cc}$ can now be integrated over the depth using Leibniz rule and boundary conditions to obtain the system linking the two variables $(\tilde{Q}, \tilde{h})$ :

$$
\begin{aligned}
& \frac{\partial \tilde{h}}{\partial \tilde{t}}+\frac{\partial}{\partial \tilde{x}} \int_{z_{b}}^{\eta} \tilde{u} d \tilde{z}=0, \\
& \frac{\partial}{\partial \tilde{t}} \int_{z_{b}}^{\eta} \tilde{u} d \tilde{z}+\frac{\partial}{\partial \tilde{x}} \int_{z_{b}}^{\eta} \tilde{u}^{2} d \tilde{z}=-\tilde{h} \frac{\partial \tilde{h}}{\partial \tilde{x}}-\tilde{h} \frac{\partial \tilde{z}_{b}}{\partial \tilde{x}}-\frac{1}{R e}\left(\frac{\partial \tilde{u}}{\partial \tilde{z}}\right)_{b},
\end{aligned}
$$

where we recall that $\tilde{h}=\tilde{\eta}-\tilde{z}_{b}$. The mass flow rate is then

$$
\tilde{Q}=\int_{\tilde{z}_{b}}^{\tilde{\eta}} \tilde{u} d \tilde{z}
$$

Thus, a closed 2D problem has been transformed into a not-closed 1-D problem. Therefore, an hypothesis on the shape of the profile is required to obtain a relation between the unknowns $\left(\int_{\tilde{z}_{b}}^{\tilde{n}} \tilde{u}^{2} d \tilde{z}\right.$, $\left.\left.\frac{\partial \tilde{u}}{\partial \tilde{z}}\right|_{b}\right)$ and the variables $(\tilde{Q}, \tilde{h})$. This allows to close the problem. Let us define $\tilde{\tau}_{b}$ the bottom stress, or wall shear stress, and $\Gamma$ the shape factor coefficient, or Boussinesq coefficient as:

$$
\tilde{\tau}_{b}=\frac{1}{R e}\left(\frac{\partial \tilde{u}}{\partial \tilde{z}}\right)_{b}, \quad \Gamma=\frac{\tilde{h} \int \tilde{u}^{2} d \tilde{z}}{\left(\int \tilde{u} d \tilde{z}\right)^{2}} .
$$

In general, these quantities are functions of $x$, where the integral $\int \cdot d \tilde{z}$ is a short hand for integration from the bottom to the free surface. The main hypothesis for Saint-Venant models is to suppose that the velocity profile has always the same "shape" in the longitudinal direction $\tilde{x}$, so that $\Gamma$ is supposed to be constant. The previous equations then read

$$
\begin{aligned}
& \frac{\partial \tilde{h}}{\partial \tilde{t}}+\frac{\partial \tilde{Q}}{\partial \tilde{x}}=0 \\
& \frac{\partial \tilde{Q}}{\partial \tilde{t}}+\frac{\partial}{\partial \tilde{x}}\left(\Gamma \frac{\tilde{Q}^{2}}{\tilde{h}}+\frac{\tilde{h}^{2}}{2}\right)=-\tilde{h} \frac{\partial \tilde{z}_{b}}{\partial \tilde{x}}-\tilde{\tau}_{b}
\end{aligned}
$$

in which $\tilde{\tau}_{b}$ has still to be written as a function of $(\tilde{Q}, \tilde{h})$ and $\Gamma$ is a constant. If one considers a steady viscous homogeneous flow in $\tilde{x}$ with a constant pressure gradient, the solution of (5a)- (5c) is a half-Poiseuille (Nusselt film solution): the shape is $\tilde{u}_{p}=\frac{3}{2} \tilde{z}(2-\tilde{z})$. This profile has the following characteristics: $\int_{0}^{1} \tilde{u}_{p} d \tilde{z}=1$ and 
$\int_{0}^{1} \tilde{u}_{p}^{2} d \tilde{z}=\frac{6}{5}$, and the slope at the wall is $\partial \tilde{u}_{p} /\left.\partial \tilde{z}\right|_{0}=3$. This gives the final closure (Boussinesq and friction coefficients) for laminar flows:

$$
\Gamma=\frac{6}{5} \text { and } \tilde{\tau}_{b}=\frac{3}{R e} \frac{\tilde{Q}}{\tilde{h}^{2}} .
$$

For turbulent flows, an heuristic approach is necessary. In this framework, equations have to be meant as statistical ones, and hence $\tilde{Q}, \tilde{h}$ represent the statistical averages over many realisations of the flow [50]. Moreover, since the higher the Re number the flatter the velocity profile, it is usually assumed to be a simple plug-flow, which corresponds to $\Gamma=1$. Furthermore, following Prandtl analysis, the friction is taken to be proportional to the square of the mean velocity $(\tilde{Q} / \tilde{h})$ with a coefficient $c_{f} / 2$ proportional to $R e^{-1 / 4}$ (and maybe function of the bottom rugosity, see Schlichting's book [49]). This gives the following closure for turbulent flows:

$$
\Gamma=1 \text { and } \tilde{\tau}_{b}=\frac{c_{f}}{2} \frac{\tilde{Q}^{2}}{\tilde{h}^{2}},
$$

(see [51] for an example with a transition from one to the other model in the Shallow Water approximation).

This kind of closure deserves a critical assessment. In particular, the hypothesis underlying these closures cannot be general. For instance, Watson 28 found a laminar self-similar solution of (5a)-(5b)-(5c) with no pressure gradient (steady flow, large velocity). This solution comes from a balance between inertia and viscosity only, it gives a linear profile in $x$ for $h$ and a velocity profile. The associated closure is:

$$
\Gamma=1.25697 \text { and } \tilde{\tau}_{b}=\frac{2.2799}{R e} \frac{\tilde{Q}}{\tilde{h}^{2}} .
$$

This shows clearly that, in general, it is necessary to solve eqs. $5 \mathrm{5a}-(5 \mathrm{c})$ to directly compute the correct coefficients $\Gamma$ and $\tilde{\tau}_{b}$.

\section{Multilayer Strategy}

\subsection{Motivation}

The proposed system (5a)-(5b)-(5c) with its boundary and initial conditions can be written back with dimensions:

$$
\begin{aligned}
\frac{\partial u}{\partial x}+\frac{\partial w}{\partial z} & =0 \\
\frac{\partial u}{\partial t}+\frac{\partial u^{2}}{\partial x}+\frac{\partial u w}{\partial z} & =-g \frac{\partial h}{\partial x}-g \frac{\partial z_{b}}{\partial x}+\frac{\partial}{\partial z}\left(\nu \frac{\partial u}{\partial z}\right),
\end{aligned}
$$

where we have dropped the hydrostatic pressure equation. Note that the viscosity $\nu$ is in fact not necessarily a constant $\nu_{0}$ (which is the case for Newtonian flows) but may be a function of the fields for non-Newtonian flows. For example for Bingham flows with a yield stress $\tau_{0}$, the equivalent viscosity is variable and is such that:

$$
\nu=\left(\frac{\left(\tau_{0} / \rho\right)}{\frac{\partial u}{\partial z}}+\nu_{0}\right) .
$$

For granular $\mu(I)$ flows [38, 20, 37] (see details on definition of $\mu(I)$ there):

$$
\nu=\left(\frac{(\mu(I) p / \rho)}{\frac{\partial u}{\partial z}}\right) .
$$

For a turbulent flow a turbulent viscosity may be used [52], for example a Prandtl mixing length model [49]:

$$
\nu=\nu_{0}+\kappa z^{2} \frac{\partial u}{\partial z},
$$

with $\kappa=0.41$ the Von Kármán constant. It is straightforward to generalise to any other viscous nonNewtonian laws, for example Herschel-Bulkley or power laws [3, 37. 


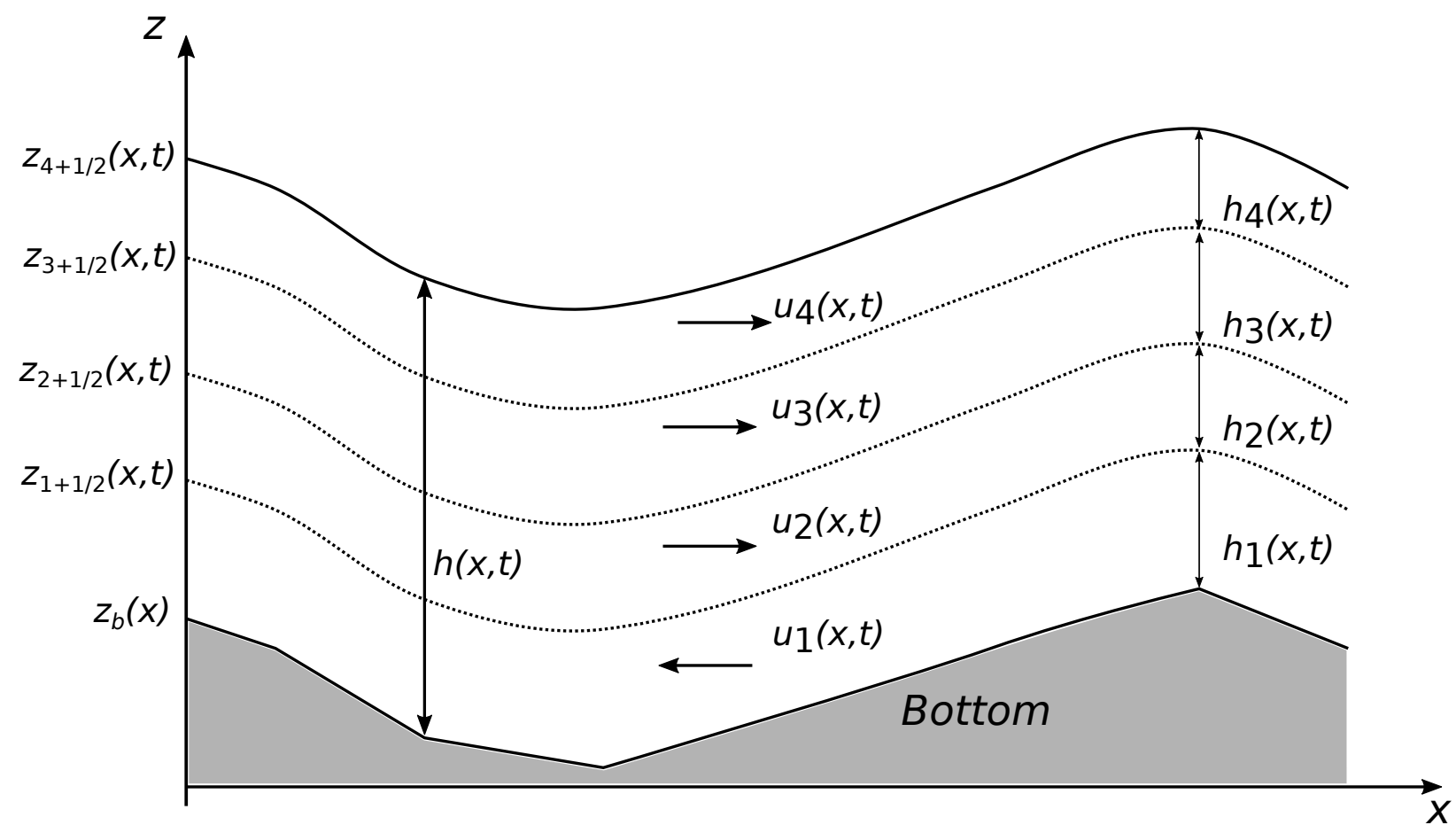

Figure 1: Sketch of the multilayer discretization, $N$ superimposed layers of thickness $h_{\alpha}$ with the horizontal velocity $u_{\alpha}$. Figure adapted from [13].

\subsection{Interpretation as a superposition of layers}

System (12a)- $12 \mathrm{~b}$ ) with the boundary conditions (3) is then discretized using the ideas of Audusse et al. [12, 13. The total fluid depth is divided in $N$ superposed layers each with its own height $h_{\alpha}$ and vertically averaged horizontal velocity $u_{\alpha}$. Each layer is advected by the flow with mass exchange across the layers as shown in figure 1. The height $h_{\alpha}$ is a fraction of the total height $h$ :

$$
l_{\alpha} h=h_{\alpha} \quad \text { with } \quad \sum_{\alpha=1}^{N} l_{\alpha}=1, \quad \text { so that } \quad h=\sum_{\alpha=1}^{N} h_{\alpha} .
$$

The vertical position of the interface between the layers is given by:

$$
z_{\alpha+1 / 2}(x, t)=z_{b}+\sum_{j=1}^{\alpha} h_{j}(x, t)
$$

and the mean horizontal velocity in each layer $u_{\alpha}$ is defined as:

$$
u_{\alpha}(x, t)=\frac{1}{h_{\alpha}} \int_{z_{\alpha-1 / 2}}^{z_{\alpha+1 / 2}} u(x, z, t) d z
$$

where $u_{\alpha+1 / 2}=u\left(x, z_{\alpha+1 / 2}, t\right)$ denotes the velocity at each interface. This discretization has been further extended in 13 where only the total height $h$ is advected by the flow allowing mass exchanges between 
layers. The complete multilayer version of equations $12 \mathrm{a} 12 \mathrm{~b}$ is then

$$
\begin{aligned}
& \frac{\partial h}{\partial t}+\sum_{\alpha=1}^{N} \frac{\partial\left(h_{\alpha} u_{\alpha}\right)}{\partial x}=0, \\
& \frac{\partial\left(h_{\alpha} u_{\alpha}\right)}{\partial t}+\frac{\partial}{\partial x}\left(h_{\alpha} u_{\alpha}^{2}+\frac{g}{2} l_{\alpha} h^{2}\right)=-g h_{\alpha} \frac{\partial z_{b}}{\partial x}+u_{\alpha+1 / 2} G_{\alpha+1 / 2}+ \\
& -u_{\alpha-1 / 2} G_{\alpha-1 / 2}+\frac{2 \nu_{\alpha}}{l_{\alpha+1}+l_{\alpha}} \frac{u_{\alpha+1}-u_{\alpha}}{h}-\frac{2 \nu_{\alpha-1}}{l_{\alpha}+l_{\alpha-1}} \frac{u_{\alpha}-u_{\alpha-1}}{h}
\end{aligned}
$$

where $G_{\alpha \pm 1 / 2}$ are the mass transfers from layer $\alpha$ to $\alpha+1$ and $\alpha-1$ respectively. The last two terms in equation (14b) represent the friction between the layers, with $\nu_{\alpha}$ the mean value of viscosity in this layer. In [13] this was presented as an ad hoc friction between layers, here it is the effective finite volume discretization of the gradient of the stress $\frac{\partial}{\partial z}\left(\nu \frac{\partial u}{\partial z}\right)$. Moreover, there is no need for a shape factor as each layer is characterised by its mean velocity, which is supposed "flat" in the finite volume discretization, $\Gamma_{\alpha}=1$. The mass term $G_{\alpha+1 / 2}$ expresses the kinematic condition at the interface $z_{\alpha+1 / 2}$ of layer $\alpha$ :

$$
G_{\alpha+1 / 2}=\frac{\partial z_{\alpha+1 / 2}}{\partial t}+u_{\alpha+1 / 2} \frac{\partial z_{\alpha+1 / 2}}{\partial x}-w\left(x, z_{\alpha+1 / 2}, t\right)
$$

and provides the mass flux leaving or entering the layer through the interface. The horizontal velocity of the interface $u_{\alpha+1 / 2}$ is computed using upwinding:

$$
\begin{cases}u_{\alpha+1 / 2}=u_{\alpha} & \text { if } \quad G_{\alpha+1 / 2}<0 \\ u_{\alpha+1 / 2}=u_{\alpha+1} & \text { if } \quad G_{\alpha+1 / 2} \geq 0\end{cases}
$$

We remark here that there is an error in sign in the original paper that presents this scheme [13. Notice that the term $G_{\alpha+1 / 2}$ can be computed also as [13]:

$$
G_{\alpha+1 / 2}=\sum_{j=1}^{\alpha}\left(\frac{\partial h_{j} u_{j}}{\partial x}-l_{j} \sum_{p=1}^{N} \frac{\partial h_{p} u_{p}}{\partial x}\right) .
$$

As a general comment, while originally this scheme was proposed to represent several shallow-water layers interacting with each other with ad-hoc friction terms, here we consider it as a natural finite-volume discretization of the 2-D reduced Navier-Stokes equations (12a)- $12 \mathrm{~b}$.

\subsection{Numerical scheme}

Equations $14 \mathrm{a}$ and $14 \mathrm{~b}$ can be seen as a system of equations of the form:

$$
\frac{\partial \mathbf{X}}{\partial t}+\frac{\partial \mathbf{F}(\mathbf{x})}{\partial x}=\mathbf{S}_{\mathbf{b}}(\mathbf{X})+\mathbf{S}_{\mathbf{e}}(\mathbf{X})+\mathbf{S}_{\mathbf{v}}(\mathbf{X})
$$

with $\mathbf{X}=\left[h, h_{1} u_{1}, h_{2} u_{2}, \ldots, h_{N} u_{N}\right]^{T}, \mathbf{F}(\mathbf{x})$ the flux, $\mathbf{S}_{\mathbf{b}}(\mathbf{X})$ the topographic source term, $\mathbf{S}_{\mathbf{e}}(\mathbf{X})$ the mass transfer in the vertical direction and $\mathbf{S}_{\mathbf{v}}(\mathbf{X})$ the viscous term. The solution procedure consists of two steps: one explicit, in which the topographic source term and the mass transfer are computed, and one implicit in which the viscous part is solved:

$$
\begin{aligned}
& \frac{\tilde{\mathbf{X}}^{n+1}-\mathbf{X}^{n}}{\Delta t}+\frac{\mathbf{F}_{i+1 / 2}^{n}-\mathbf{F}_{i-1 / 2}^{n}}{\Delta x}=\mathbf{S}_{\mathbf{b}}\left(\mathbf{X}^{n}\right)+\mathbf{S}_{\mathbf{e}}\left(\mathbf{X}^{n}\right) \\
& \frac{\mathbf{X}^{n+1}-\tilde{\mathbf{X}}^{n+1}}{\Delta t}=\mathbf{S}_{\mathbf{v}}\left(\mathbf{X}^{n+1}\right)
\end{aligned}
$$

with $n$ the time step and $i$ the index of the cell. 


\subsubsection{Explicit Step}

In the explicit step the fluxes $\mathbf{F}$, the source term $\mathbf{S}_{b}$ and the mass term $\mathbf{S}_{e}$ are evaluated. The fluxes are computed solving a Riemann problem. The procedure consists in computing the gradient of the primary fields $h, \mathbf{u}$ and $z$, reconstructing the left and right states and applying a Riemann solver, e.g. [53. For the topographic term the hydrostatic reconstruction presented in [54] is used:

$$
S b_{\alpha, i}^{n}=l_{\alpha}\left(\frac{g}{2}\left(h_{i+1 / 2-}^{n}\right)^{2}-\frac{g}{2}\left(h_{i-1 / 2+}^{2}\right)^{2}\right)
$$

with the following re-estimated values:

$$
\begin{aligned}
h_{i+1 / 2-}^{n} & =h_{i}^{n}+z_{b, i}-z_{b, i+1 / 2}, \\
h_{i+1 / 2+}^{n} & =h_{i}^{n}+z_{b, i+1}-z_{b, i+1 / 2}, \\
z_{b, i+1 / 2} & =\max \left\{z_{b, i}, z_{b, i+1}\right\} .
\end{aligned}
$$

In 54 it has been shown that this discretization of the source term preserves the steady state given by a "lake at rest". Once the fluxes $\mathbf{F}$ are known it is possible to compute the mass fluxes between the layers $G_{\alpha+1 / 2}$ from equation (16). Then it is possible to compute the vertical velocity from the definition of the mass flux (equation (15))

$$
w\left(x, z_{\alpha+1 / 2}\right)=\frac{\partial z_{\alpha+1 / 2}}{\partial t}-G_{\alpha+1 / 2}+u_{\alpha+1 / 2} \frac{\partial z_{\alpha+1 / 2}}{\partial x}
$$

which combined with equation 13 gives

$$
w\left(x, z_{\alpha+1 / 2}\right)=\frac{\partial h}{\partial t} \sum_{j=0}^{\alpha} l_{j}-G_{\alpha+1 / 2}+u_{\alpha+1 / 2}\left[\frac{\partial z_{b}}{\partial x}+\frac{\partial h}{\partial x} \sum_{j=0}^{\alpha} l_{j}\right],
$$

for the final expression of the transverse velocity.

\subsubsection{Viscous Step}

The final output vector is computed starting from the output of the explicit step and adding the viscous term:

$$
\mathbf{X}^{n+1}=\tilde{\mathbf{X}}^{n+1}+\Delta t \mathbf{S}_{\mathbf{v}} .
$$

The system (18) is composed of $N$ equations of the type

$$
h_{\alpha}^{n+1} u_{\alpha}^{n+1}=h_{\alpha}^{n+1} \tilde{u}_{\alpha}^{n+1}+\frac{2 \Delta t^{n} \nu_{\alpha}}{h^{n+1}} \frac{u_{\alpha+1}^{n+1}-u_{\alpha}^{n+1}}{l_{\alpha+1}+l_{\alpha}}-\frac{2 \Delta t^{n} \nu_{\alpha-1}}{h^{n+1}} \frac{u_{\alpha}^{n+1}-u_{\alpha-1}^{n+1}}{l_{\alpha}+l_{\alpha-1}}
$$

and the solution is obtained by solving a trilinear system $N \mathrm{x} N$. Note that the viscous step does not modify $h$. Equation 19 can be written as:

$$
\begin{gathered}
-u_{\alpha-1}^{n+1} \frac{2 \Delta t^{n} \nu_{\alpha-1}}{\left(l_{\alpha}+l_{\alpha-1}\right) h^{n+1}}+u_{\alpha}^{n+1}\left[h_{\alpha}^{n+1}+\frac{2 \Delta t^{n} \nu_{\alpha}}{\left(l_{\alpha+1}+l_{\alpha}\right) h^{n+1}}+\right. \\
\left.+\frac{2 \Delta t^{n} \nu_{\alpha-1}}{\left(l_{\alpha}+l_{\alpha-1}\right) h^{n+1}}\right]-u_{\alpha+1}^{n+1} \frac{2 \Delta t^{n} \nu_{\alpha}}{\left(l_{\alpha+1}+l_{\alpha}\right) h^{n+1}}=h_{\alpha}^{n+1} \tilde{u}_{\alpha}^{n+1}
\end{gathered}
$$

which is equivalent to:

$$
A_{\alpha}^{n+1} u_{\alpha-1}^{n+1}+B_{\alpha}^{n+1} u_{\alpha}^{n+1}+C_{\alpha}^{n+1} u_{\alpha+1}^{n+1}=D_{\alpha}^{n+1} .
$$


The coefficients $A_{\alpha}, B_{\alpha}, C_{\alpha}$ and $D_{\alpha}$ are equal to

$$
\begin{cases}A_{\alpha}^{n+1}=-\frac{2 \Delta t^{n} \nu_{\alpha-1}}{\left(l_{\alpha}+l_{\alpha-1}\right) h^{n+1}} & \\ B_{\alpha}^{n+1}=h_{\alpha}^{n+1}+\frac{2 \Delta t^{n} \nu_{\alpha}}{\left(l_{\alpha+1}+l_{\alpha}\right) h^{n+1}}+\frac{2 \Delta t^{n} \nu_{\alpha-1}}{\left(l_{\alpha}+l_{\alpha-1}\right) h^{n+1}} & \\ C_{\alpha}^{n+1}=-\frac{2 \Delta t^{n} \nu_{\alpha}}{\left(l_{\alpha+1}+l_{\alpha}\right) h^{n+1}} & \alpha=2, \ldots, N-1 \\ D_{\alpha}^{n+1}=\tilde{q}_{\alpha}^{n+1} & \alpha=2, N \text { is }\end{cases}
$$

in all the intermediate layers whereas for the first and the last layer it is necessary to add boundary conditions to close the system for the viscous stress, as discussed in the next section. With this notation the system (18) can be written in matrix form as:

$$
\left[\begin{array}{ccccc}
B_{1}^{n+1} & C_{1}^{n+1} & & & \\
A_{2}^{n+1} & B_{2}^{n+1} & C_{2}^{n+1} & & \\
& & \cdots & & \\
& & A_{N-1}^{n+1} & B_{N-1}^{n+1} & C_{N-1}^{n+1} \\
& & & A_{N}^{n+1} & B_{N}^{n+1}
\end{array}\right]\left[\begin{array}{c}
u_{1}^{n+1} \\
u_{2}^{n+1} \\
\vdots \\
u_{N-1}^{n+1} \\
u_{N}^{n+1}
\end{array}\right]=\left[\begin{array}{c}
D_{1}^{n+1} \\
D_{2}^{n+1} \\
\vdots \\
D_{N-1}^{n+1} \\
D_{N}^{n+1}
\end{array}\right]
$$

and using the Thomas algorithm it is possible to compute the vector $\mathbf{X}^{n+1}$ and get the solution at time step $n+1$, provided the value of $A_{\alpha}^{n+1}, B_{\alpha}^{n+1} C_{\alpha}^{n+1}, D_{\alpha}^{n+1}$ for $\alpha=1$ and $N$ are given, as described below.

\subsubsection{Boundary conditions}

On the top layer a zero Neumann condition is imposed (free-slip condition on the top boundary), while on the bottom layer a no-slip boundary condition is imposed. This may be generalised as:

$$
\frac{\partial u_{N+1 / 2}}{\partial z}=\dot{u}_{t}
$$

and imposing a Navier slip (Robin, or mixed) condition at the bottom:

$$
u_{1 / 2}=u_{b}+\lambda_{b} \frac{\partial u_{1 / 2}}{\partial z}
$$

In the following we will consider a free-slip condition on the top boundary i.e. $\dot{u}_{t}=0$, and a no-slip condition on the bottom boundary i.e. $u_{b}=0$ and $\lambda_{b}=0$. We now consider equation 19 for layer $N$

$$
h_{N}^{n+1} u_{N}^{n+1}=h_{N}^{n+1} \tilde{u}_{N}^{n+1}+\frac{2 \Delta t^{n} \nu_{N}}{h^{n+1}} \frac{u_{N+1}^{n+1}-u_{N}^{n+1}}{l_{N+1}+l_{N}}-\frac{2 \Delta t^{n} \nu_{N-1}}{h^{n+1}} \frac{u_{N}^{n+1}-u_{N-1}^{n+1}}{l_{N}+l_{N-1}}
$$

Layer $N+1$ is a ghost layer used to impose the boundary condition on the top layer (the same is done for the bottom layer). Because the ghost layer has the same thickness as the top layer i.e. $l_{N+1}=l_{N}$, from equation 20 it follows that

$$
u_{N+1}=u_{N}+\dot{u}_{t} h_{N}=u_{N}+\dot{u}_{t} l_{N} h
$$

which, substituted in equation 22 , gives the coefficients:

$$
\left\{\begin{array}{l}
A_{N}^{n+1}=-\frac{2 \Delta t^{n} \nu_{N-1}}{\left(l_{N}+l_{N-1}\right) h^{n+1}} \\
B_{N}^{n+1}=h_{N}^{n+1}+\frac{2 \Delta t^{n} \nu_{N-1}}{\left(l_{N}+l_{N-1}\right) h^{n+1}} \\
C_{N}^{n+1}=0 \\
D_{N}^{n+1}=\tilde{q}_{N}^{n+1}+\Delta t^{n} \nu_{N} \dot{u}_{t}
\end{array}\right.
$$


The equation for the first layer is $(\alpha=1$ in equation 190$)$

$$
h_{1}^{n+1} u_{1}^{n+1}=h_{1}^{n+1} \tilde{u}_{1}^{n+1}+\frac{2 \Delta t^{n} \nu_{1}}{h^{n+1}} \frac{u_{2}^{n+1}-u_{1}^{n+1}}{l_{2}+l_{1}}-\frac{2 \Delta t^{n} \nu_{0}}{h^{n+1}} \frac{u_{1}^{n+1}-u_{0}^{n+1}}{l_{1}+l_{0}}
$$

and from the Navier slip boundary condition 21 it is possible to express the velocity $u_{0}$ as

$$
u_{0}=\frac{2 h_{1}}{2 \lambda_{b}+h_{1}} u_{b}+\frac{2 \lambda_{b}-h_{1}}{2 \lambda_{b}+h_{1}} u_{1}
$$

which substituted in $(23)$ gives the coefficients

$$
\left\{\begin{array}{l}
A_{1}^{n+1}=0 \\
B_{1}^{n+1}=h_{1}^{n+1}+\frac{2 \Delta t^{n} \nu_{1}}{\left(l_{2}+l_{1}\right) h^{n+1}}+\frac{2 \Delta t^{n} \nu_{1}}{2 \lambda_{b}+l_{1} h^{n+1}} \\
C_{1}^{n+1}=-\frac{2 \Delta t^{n} \nu_{2}}{\left(l_{2}+l_{1}\right) h^{n+1}} \\
D_{1}^{n+1}=\tilde{q}_{1}^{n+1}+u_{b} \frac{2 \Delta t^{n} \nu_{1}}{2 \lambda_{b}+l_{1} h^{n+1}} .
\end{array}\right.
$$

Hence, the values of $A_{\alpha}^{n+1}, B_{\alpha}^{n+1} C_{\alpha}^{n+1}$ and $D_{\alpha}^{n+1}$ for $\alpha=1$ and $N$ are indeed obtained.

\subsection{4. "Standard" Shallow-Water limit}

Of course, with only one layer $N=1$ the same algorithm corresponds to the standard Shallow Layer equations (11a)- $111 \mathrm{~b})$. The shape factor $\Gamma_{1}$ is indeed equal to one. The sole difference lies in the viscous step which is written in a semi implicit way :

$$
u_{1}^{n+1}=\frac{u_{1}^{n}}{1+3 \nu_{1} \Delta t^{n} /\left(h_{1}^{n}\right)^{2}}
$$

for laminar flows, but any other friction law may be easily adapted.

\subsection{Stability and scheme properties}

The stability condition for the multilayer Saint-Venant equations needs to ensure that the water leaving a cell in a timestep is smaller than the actual value of the depth [13]

$$
\Delta t^{n} \leq \min \left\{\frac{l_{\alpha} H_{i}^{n} \Delta x_{i}}{l_{\alpha} H_{i}^{n}\left(\left|u_{\alpha, i}^{n}\right|\right)+\Delta x_{i}\left(\left[G_{\alpha+1 / 2, i}^{n+1 / 2}\right]_{-}+\left[G_{\alpha-1 / 2, i}^{n+1 / 2}\right]_{+}\right)}\right\}
$$

with $\alpha$ going from 1 to the number of layers and $i$ going from one to the number of points in the x-coordinate. In our implementation, we have not applied any modification to the scheme, but we have introduced an adequate boundary condition to handle the bottom friction. For this reason our implementation preserves all the properties of the scheme already demonstrated for the original one [54, 13.

\section{Results}

Having presented the Boundary-Layer model and the numerical scheme, this section is devoted to illustrating some numerical applications. These examples are used both to validate the method and to point out the differences with the "standard" Saint-Venant approach. In particular, the impact of the shape factor and friction coefficient which are to be closed in shallow-water approximation, are assessed. First the viscous examples of slumps by Huppert [55] and [56] are considered. In these cases, the flow is so viscous that the velocity remains always a half-Poiseuille one and the inertia is negligible in (11a)-(11b). Non-linearity is introduced afterwards in the standing jump cases [25]. Web links for the codes of most of the examples presented here are given in the appendix. Among them one of the example of [11, 12, 13, is reproduced. 

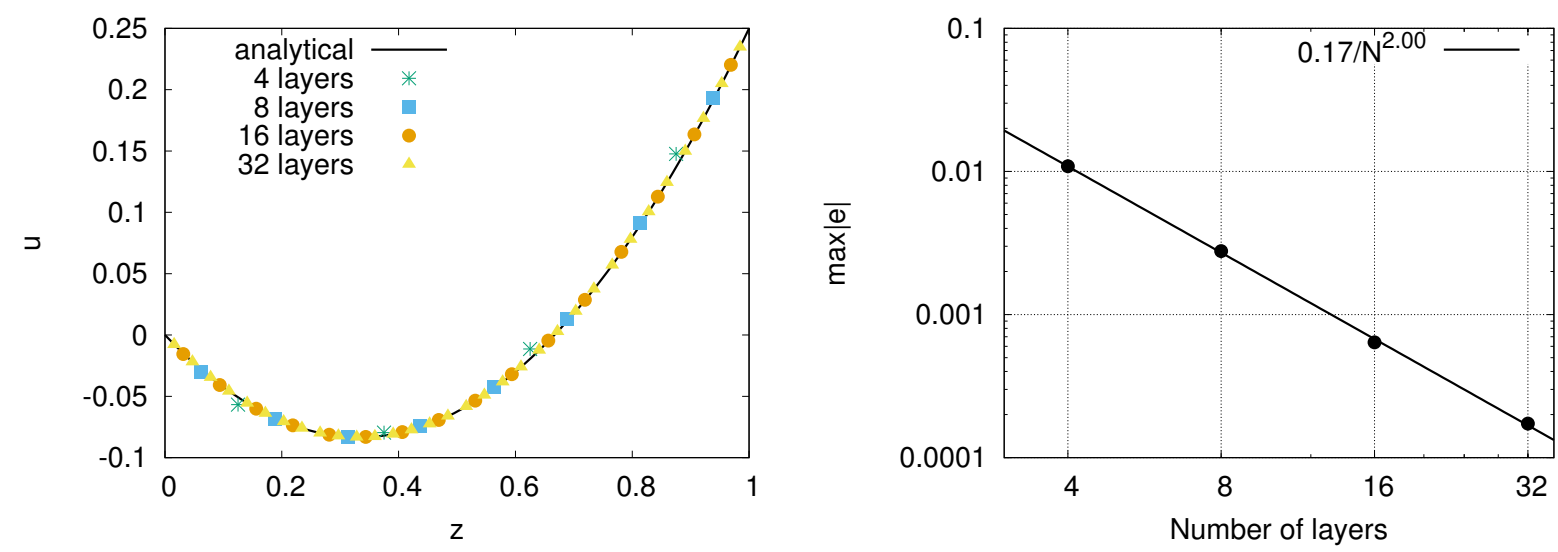

Figure 2: Comparison between analytical solution and numerical solution (left); order of convergence (right).

\subsection{Stress induced flow}

To validate our implementation we consider the flow of a fluid in a closed basin driven by a constant wind stress at the top (wind-driven cavity, as proposed in [57]). The action of the wind induces a stress on the free surface which causes the motion of the liquid. The value of the wind stress gives the scale of the flow. Because the fluid is confined, the only stationary solution is a steady-state recirculation inside the basin. The boundary conditions on the horizontal velocity are Neumann on the top and no-slip on the other sides. The solution for the vertical profile of the horizontal velocity at the centerline is, by symmetry:

$$
\tilde{u}=\frac{\tilde{z}}{4}(3 \tilde{z}-2)
$$

so that the stress at the surface is unity and the mass flow is zero. If the domain is long enough this solution is valid for a large part of the flow, except at the boundaries (left and right). We report in figure 2 (left) the comparison between results obtained with our solver and the analytical solution. We vary the number of layers from 4 to 32 and keep constant the horizontal resolution to 64 grid cells. We compute the norm $L_{1}$ of the error and verify that the use of the boundary condition 23) gives a second-order convergence rate while in [13] it is reported that the use of a Navier friction coefficient for the bottom condition reduce the convergence rate order from 2 to 1.7 .

\subsection{Viscous collapse on a plate}

\subsubsection{Horizontal plate}

The slump of an initial heap of viscous fluid on a horizontal plate is considered. This is a double dam break viscous problem. In this flat bottom case $([55]), \tilde{z}_{b}=0$, the pressure gradient balances friction so that from $11 \mathrm{~b}$ one obtains $\tilde{Q}$, which is substituted in mass conservation $11 \mathrm{a}$, and the laminar Saint-Venant equations simplify into a single evolution equation (with $k=\frac{R e}{3}$ ):

$$
\frac{\partial \tilde{h}}{\partial \tilde{t}}-k \frac{\partial}{\partial \tilde{x}}\left(\tilde{h}^{3} \frac{\partial \tilde{h}}{\partial \tilde{x}}\right)=0
$$

This equation has a self-similar solution $\tilde{h}=\tilde{t}^{-1 / 5} \mathcal{H}\left(\tilde{x} \tilde{t}^{-1 / 5}\right)$ of the self-similar variable $\eta=\tilde{x} \tilde{t}^{-1 / 5}$ which turns out to be:

$$
\mathcal{H}(\eta)=\frac{3^{2 / 3} \eta_{f}^{2 / 3}}{10^{1 / 3}}\left(1-\frac{\eta^{2}}{\eta_{f}^{2}}\right)^{1 / 3} \quad \text { where } \eta_{f}=\frac{2^{1 / 5} 5^{4 / 5} \Gamma\left(\frac{5}{6}\right)^{3 / 5}}{3^{2 / 5} \pi^{3 / 10} \Gamma\left(\frac{1}{3}\right)^{3 / 5}}
$$

with $\Gamma$ the Euler function, not to be confused with the Boussinesq coefficient. On figure 3 an example of the full resolution of $12 \mathrm{a}-(12 \mathrm{~b})$ is presented, showing some profiles of $\tilde{h}(\tilde{x}, \tilde{t})$ during the collapse. The 

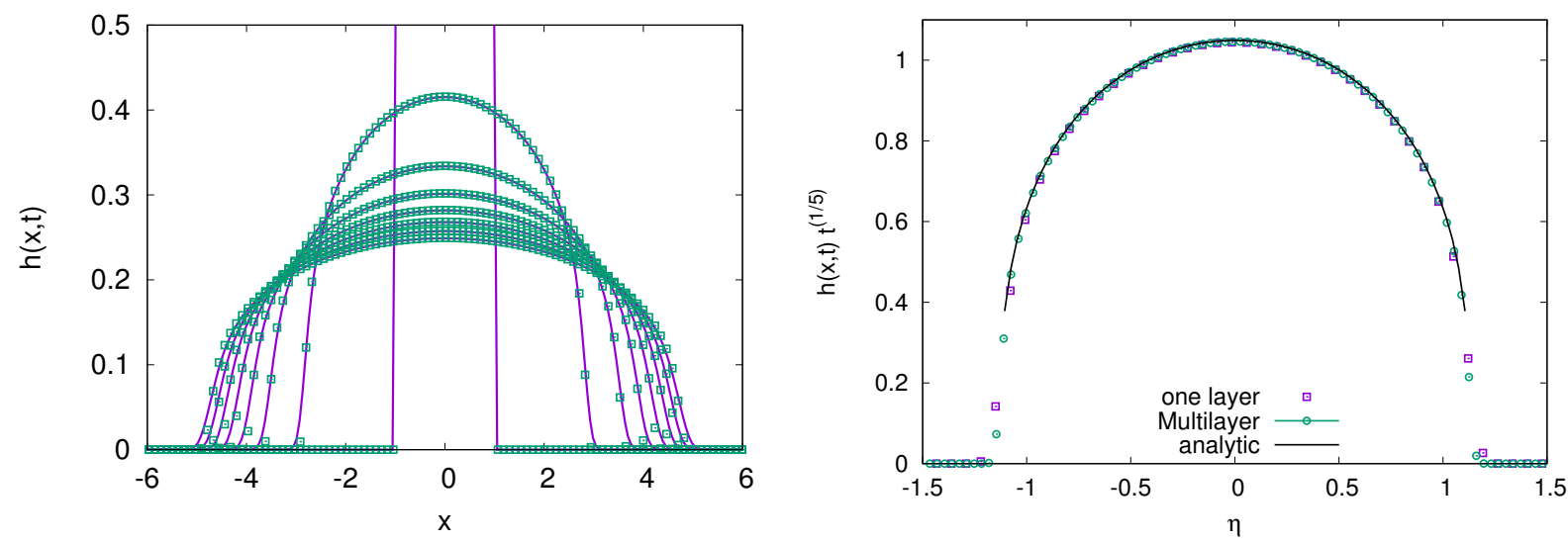

Figure 3: Collapse of a viscous flow on a flat surface. Left: at $\tilde{t}=100,300,500 \ldots 1500$ plot of $\tilde{h}(\tilde{x}, \tilde{t})$ for Saint-Venant (solid purple line) and multilayer resolution (green $\square)$. The initial height is $\tilde{h}(\tilde{x}, 0)=1$ for $-1<\tilde{x}<1$, and surface $\int_{0}^{1} h(\tilde{x}, 0) d \tilde{x}=2$. Right: plot for $\tilde{t}>500$ of $\mathcal{H}(\eta)=\tilde{t}^{1 / 5} \tilde{h}(\tilde{x}, \tilde{t})$ as function of $\eta=\tilde{x} / \tilde{t}^{1 / 5}$ with Saint-Venant (purple $\square$ ) and multilayer resolution (green $\circ$ ), and analytical (solid black line), which is here numerically $\left(0.9\left(1.28338-\eta^{2}\right)\right)^{1 / 3}$.

same curves are plotted in self-similar variables demonstrating the collapse of all the rescaled heights on the master curve $\mathcal{H}(\eta)$ with the self-similar variable $\eta$. The solution of $(11 \mathrm{a})-(11 \mathrm{~b})$ gives almost the same result, as well as the direct resolution of Eq. (26) (not presented here). As expected, the resolution of (12a) 12b), after a short transient phase, gives the computed values:

$$
\frac{\tilde{h} \int \tilde{u}^{2} d \tilde{z}}{\left(\int \tilde{u} d \tilde{z}\right)^{2}} \simeq 1.2 \text { and } \frac{\partial \tilde{u}}{\partial \tilde{z}} 0 \frac{\tilde{h}^{2}}{\tilde{Q}} \simeq 3.0
$$

which are the half-Poiseuille Nusselt values.

\subsubsection{Inclined plate}

An initial heap of viscous fluid is released on an inclined plate with a constant slope 56. In this case, pressure gradient and inertia are negligible, there is only a balance between the projection of gravity along the plate and the viscous friction. The laminar Saint-Venant equations simplify into a single evolution equation: $\left(k=R e \frac{\partial \tilde{z}_{b}}{\partial \tilde{x}}\right)$ :

$$
\frac{\partial \tilde{h}}{\partial \tilde{t}}-k \tilde{h}^{2} \frac{\partial \tilde{h}}{\partial \tilde{x}}=0 .
$$

This equation has a self-similar solution $\tilde{t}^{-1 / 3} \mathcal{H}\left(\tilde{x} / \tilde{t}^{1 / 3}\right)$ which turns out to be:

$$
\mathcal{H}(\eta)=\sqrt{(\eta) / k}
$$

so that for a given initial mass $A_{0}=\int_{0}^{\tilde{x}_{1}} h(\tilde{x}, 0) d \tilde{x}$, the flow spreads up to $\tilde{x}_{f}=\left(\frac{9 A_{0}^{2} k \tilde{t}}{4}\right)^{1 / 3}$ and

$$
\tilde{h}=\tilde{t}^{-1 / 3} \sqrt{\left.(\tilde{x}) \tilde{t}^{-1 / 3}\right) / k}=\sqrt{\frac{\tilde{x}}{k \tilde{t}}} .
$$

Huppert's resolution to find the solution is based on the method of characteristics. It is not based on this self-similar analysis. See on figure 4 the numerical resolution and some profiles at different times. Again, numerical resolution of $12 \mathrm{a}-\mathrm{12b}$, after a short transient phase, gives the half-Poiseuille Nusselt profile. The self-similar solution is obtained (figure 4) for large times for the Saint-Venant (eq. (11a)- (11b) and the multilayer resolution of RNSP (eq. $12 \mathrm{a}-12 \mathrm{~b}$ ). The profiles are plotted in self-similar variables showing the collapse of all the rescaled heights on the master curve $\mathcal{H}(\eta)$ with the self-similar variable $\eta$. 
Note that a small time step $\Delta t$ (small CFL condition) is needed in the Saint-Venant approximation, in 160 order to prevent an artificial numerical slip of the bump. Moreover, with the Saint-Venant model, a spurious small numerical overshoot appears at the shock, which is also present in the multilayer solution, when $N$ is small.
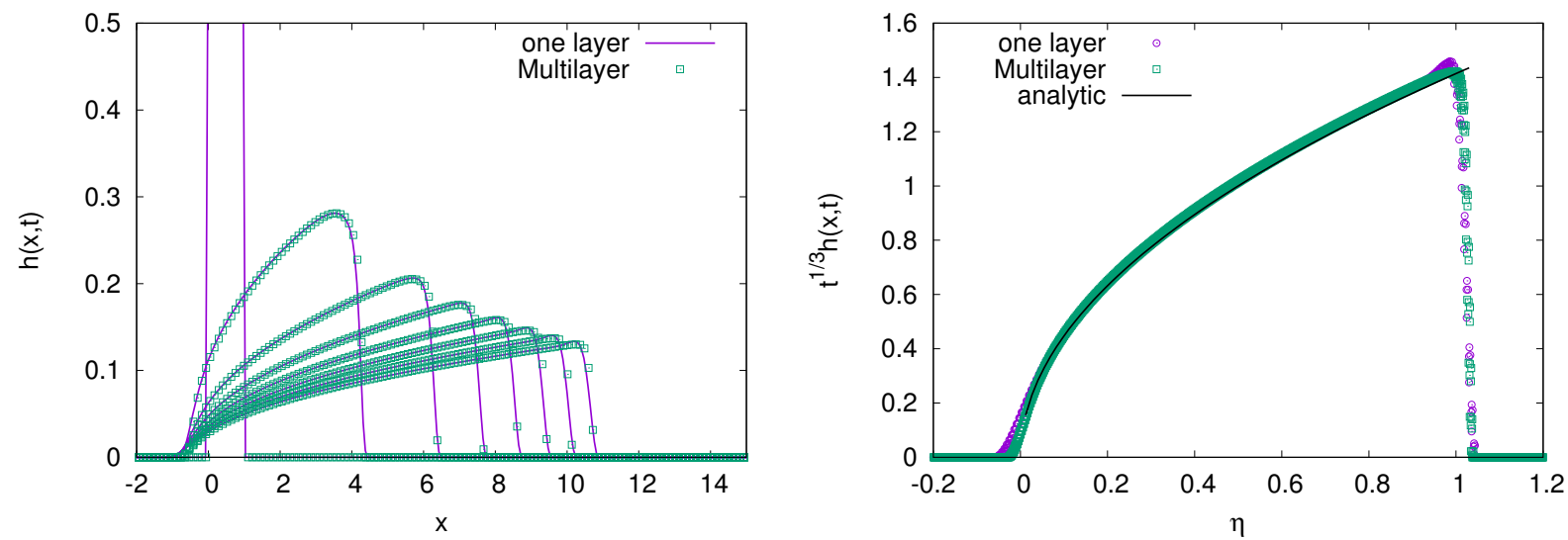

Figure 4: Collapse of a viscous flow along a slope. (left) at $\tilde{t}=100,300,500 \ldots 1500$ plot of $\tilde{h}(\tilde{x}, \tilde{t})$ for Saint-Venant (purple solid line) and multilayer resolution (empty green square). The initial height is $\tilde{h}(\tilde{x}, 0)=1$ for $0<\tilde{x}<1$, and surface $\int_{0}^{1} \tilde{h}(\tilde{x}, 0) d \tilde{x}=1$. (right) plot for $\tilde{t}>500$ of $\mathcal{H}(\eta)=\tilde{t}^{1 / 3} \tilde{h}(\tilde{x}, \tilde{t})$ as a function of $\eta=\tilde{x} / \tilde{t}^{1 / 3}$ with Saint-Venant (empty purple circle), multilayer resolution (empty green square), and analytical square root self-similar solution. Here $\alpha=1 / 2$, so that $\tilde{x}_{f}=\left(3^{2 / 3} / 2\right) \tilde{t}^{1 / 3}$, with $\left(3^{2 / 3} / 2\right) \simeq 1.04$.

\subsection{Hydraulic jumps on flat surfaces}

The previous two examples were relevant for the viscous and the topographic terms. In this section, we show the application of the proposed model to the study of a standing jump. This is a particularly interesting case where all the terms, inertia, viscosity, pressure gradient and topography are important (dominant balance).

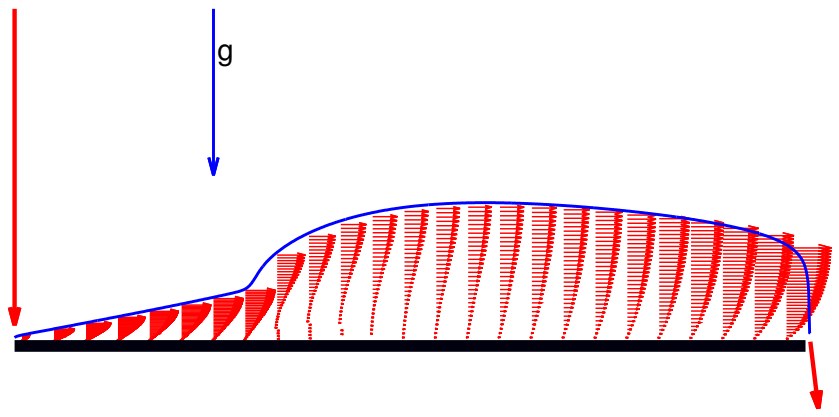

Figure 5: Sketch of the flow, the free surface is in blue, longitudinal velocity profiles in red. The fluid is falling on the left (represented by the long vertical arrow) and turns to be parallel to the plate. A thin supercritical layer grows gently. At the end of the plate, fluids falls down. A jump in the height of the free surface appears, the flow slows down across this abrupt variation.

\subsubsection{Hydraulic jump on a horizontal surface}

First the application of the proposed multilayer model is used to study a standing jump problem previously analysed by 25. The flow is sketched in figure 5 . A vertical 2D jet, not described in the thin layer approximation, with flow rate $Q_{0}$, impacts at the center of a plate of length $2 L$ (only one half is presented). 
At the beginning of the plate, the flow is very fast and supercritical. Then, due to the fact that the flat plate is of finite extent, and due to viscous effects, a deceleration occurs downstream. Hence, a jump connects a region of fast flow (supercritical) to another of slower velocity (subcritical). This decrease is due to viscosity so that for this configuration $R e=1$ which gives $L=h_{0}\left(c_{0} h_{0}\right) / \nu_{0}$. This problem has been described, for a plane surface using the steady RNSP model 25, and in the axi-symmetric case 26, yet using a different scaling of the equations. Instead of scaling velocity with $c_{0}$, Higuera uses $Q_{0} / h_{0}$ were $Q_{0}$ is the flow rate. The steady equations obtained in 25] are therefore

$$
\frac{\partial \bar{u}}{\partial \bar{x}}+\frac{\partial \bar{w}}{\partial \bar{z}}=0, \bar{u} \frac{\partial \bar{u}}{\partial \bar{x}}+\bar{w} \frac{\partial \bar{u}}{\partial \bar{z}}=-S \frac{\partial \bar{h}}{\partial \bar{x}}+\frac{\partial^{2} \bar{u}}{\partial \bar{z}^{2}} \text { given } \int_{0}^{\bar{h}} \bar{u} d \bar{z}=1,
$$

with this choice the Froude number is $S^{-1 / 2}$. With our choice, those equations are:

$$
\frac{\partial \tilde{u}}{\partial \tilde{x}}+\frac{\partial \tilde{w}}{\partial \tilde{z}}=0, \frac{\partial \tilde{u}}{\partial \tilde{t}}+\frac{\partial \tilde{u}^{2}}{\partial \tilde{x}}+\frac{\partial \tilde{u} \tilde{w}}{\partial \tilde{z}}=-\frac{\partial \tilde{h}}{\partial \tilde{x}}+\frac{\partial^{2} \tilde{u}}{\partial \tilde{z}^{2}} \text { given } \int_{0}^{\tilde{h}} \tilde{u} d \tilde{z}=\tilde{Q}
$$

It is straightforward to see that the relation between $S$ and $\tilde{Q}$ is:

$$
S^{-1 / 2}=\tilde{Q}^{5 / 2}
$$

For steady flow $\tilde{Q}$ is indeed constant, then the value of $\tilde{Q}^{5 / 2}$ is a global Froude number.

Let us begin with analysing some asymptotic behaviours, for which analytical results can be obtained. For small $S$, or large $\tilde{Q}$, the pressure gradient is negligible, from eq. 28 one obtains the Watson self-similar solution (steady flow, balance between inertia and viscosity) $u_{w}=\frac{1}{x} f(\eta)$ with $\eta=y / x$. The function $f$ is solution of the equation $f^{\prime \prime}=-f^{2}$ with $f(0)=0, f^{\prime}\left(H_{w}\right)=0$ for a unit flow rate $\int_{0}^{H_{w}} f(\eta) d \eta=1$. After solving the equation, one finds: $H_{w}=1.8138, f\left(H_{w}\right)=0.8964$ and $f^{\prime}(0)=0.693$. So that $\tilde{h}(\tilde{x})=1.8138 \tilde{x}$. The already mentioned shape factor is $\Gamma=H_{w} \frac{\int_{0}^{H_{w}} f(\eta)^{2} d \eta}{\left(\int_{0}^{H} w f(\eta) d \eta\right)^{2}}=1.25697$, the shear is $\tau_{b}=f^{\prime}(0) H_{w}^{2}=2.2799$.

Another limit of eq. 28 may be obtained for large $S$, or small $\tilde{Q}$, when the pressure gradient is no more negligible, but inertia is now negligible, one obtains the Poiseuille solution (balance between pressure gradient and viscosity).

$$
\tilde{u}=-\tilde{h}^{2} \frac{\partial \tilde{h}}{\partial \tilde{x}} \frac{(\tilde{y})}{2 \tilde{h}}\left(2-\frac{(\tilde{y})}{\tilde{h}}\right), \quad \tilde{Q}=-\frac{\tilde{h}^{3}}{3} \frac{\partial \tilde{h}}{\partial \tilde{x}}
$$

so that one can solve the equation for $\tilde{h}(\tilde{x})$, and as a small height (even 0 ) is given at the boundary condition, we neglect it and obtain as an approximation of the surface position for small flow rate near the output:

$$
\tilde{h}(\tilde{x})=(12 \tilde{Q})^{1 / 4}(1-\tilde{x})^{1 / 4},
$$

the shape factor is $\Gamma=\frac{6}{5}$ and shear is $\tilde{\tau}_{b}=3$.

We present here the numerical results for the full problem. The system of equations is solved using $\tilde{Q}$ (or $S$ ) as a parameter. A first flat profile is imposed at the input at $\tilde{x}>0$ on a small given height (say 0.1 ) compatible with Watson's solution. At the outlet a zero Neumann boundary condition is imposed on the velocity and a zero depth, $\tilde{h} \rightarrow 0$. The simulations have been performed using 256 points in the horizontal direction and 30 layers in the vertical direction. These values have been chosen checking the convergence both on the thickness of the hydraulic jump, influenced by the horizontal resolution, and on the skin friction which is affected by the number of layers.

Figure 6 shows a comparison of the free surface profile and the skin friction (resp. $\bar{h}(\bar{x})$ and $\left.\partial \bar{u} /\left.\partial \bar{z}\right|_{0}\right)$, for different values of $S$, between the solution obtained with the proposed solver, written in "bar" variables, eq. (28), and the data from 25. The agreement is quite good.

Figure 7 shows an example of the free surface height, the shape factor and the skin friction (resp. $\tilde{h}(\tilde{x})$, $\Gamma$ and $\partial \tilde{u} /\left.\partial \tilde{z}\right|_{0}$ ), for $\tilde{Q}=1$. Consistently with previous results, the numerical solution after a small entrance effect due to the flat profile boundary condition, is close to $\Gamma=1.25697$ corresponding to the shape factor 


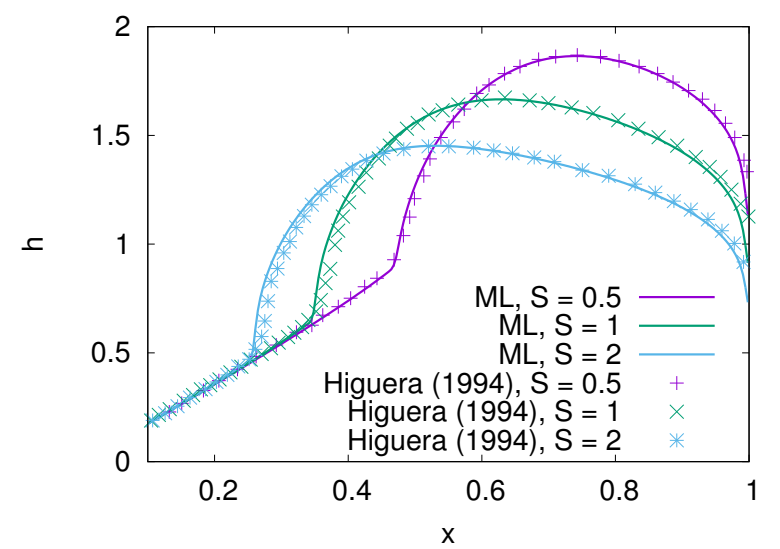

(a)

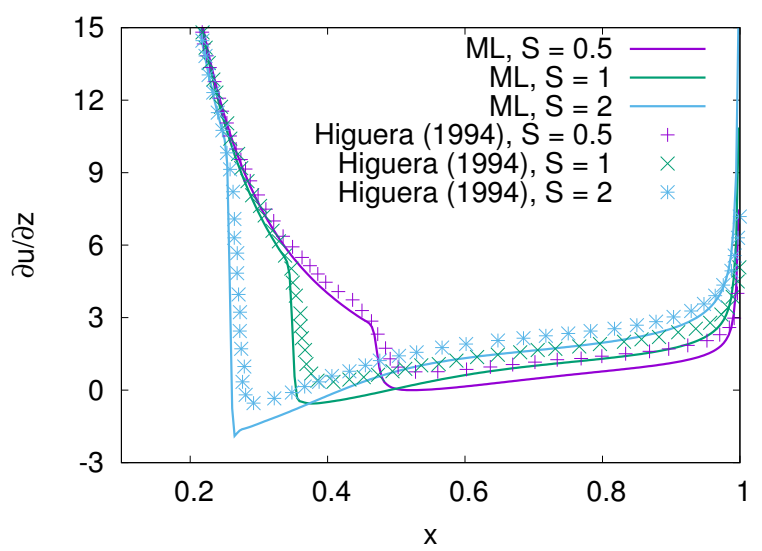

(b)

Figure 6: Comparison of the liquid depth $\bar{h}$ (a) and skin friction (b) solution of eq. 27 with the data from 25. Multilayer solver (ML) in "bar" variables (Froude number is $S^{-1 / 2}$ ): $S=0.5$ solid purple line, $S=1$ solid green line, $S=2$ solid blue line. Data from [25]: $S=0.5$ red,$+ S=1$ green $\times, S=2$ blue $*$.

value of Watson's solution. The reduced bottom stress has also a value close to the Watson one, that is 2.2799. Then $\Gamma$ increases across the jump, this corresponds to a flow separation, which is associated with a recirculation bubble with $\partial \tilde{u} /\left.\partial \tilde{z}\right|_{0}<0$ and a large value of $\tilde{h}$. Then it decreases to a value close to $\Gamma=1.2$ corresponding to the half-Poiseuille profile. This regime develops after the jump and before the end of the plate where it is accelerated and goes to 1 . The shear, after being negative in the recirculation bubble associated to the increase of $\tilde{h}$, crosses the value 3 associated to the Nusselt solution. Finally, it increases as the velocity increases at the output.

This scenario is the same for all the values of $\tilde{Q}$ : values of $\frac{\tilde{h} \int \tilde{u}^{2} d \tilde{z}}{\left(\int \tilde{u} d \tilde{z}\right)^{2}}$ and of $\left.\frac{\partial \tilde{u}}{\partial \tilde{z}}\right|_{0} \frac{\tilde{h}^{2}}{\tilde{Q}}$ are plotted for a wide range of $\tilde{Q}$ on figure 8 . Focus on $\tilde{Q}<.3$ shows that the curves collapse on $6 / 5$ (figure $8 \mathrm{a}$ ) and 3 (figure $8 \mathrm{~b}$ ), which are the values of the Poiseuille profile. Focus on $\tilde{Q}>1.3$ shows that the curves collapse on 1.25697 (figure 8a) and 2.2799 (figure 8b), which are the Watson values. Note that the shape factor is never one.

To conclude, on figure 9 the impact of the value of the friction on the results obtained via the 1-D Saint Venant model is shown. In particular, the comparison is made with the cases in which the friction term $\tau_{b}$ is: $-3 \tilde{Q} / h^{2}$ and $-2.2799 \tilde{Q} / h^{2}$. The value of $\Gamma$ is set to one. One can see that in $1-\mathrm{D}$ Shallow Water theory, the jump is very sharp. The position of the jump is delayed with Watson's friction estimation, when compared to Poiseuille's. The shear stress is always positive, though small. For the sake of comparison, the complete BL solution is plotted as well. One can appreciate the main differences: (i) the jump is smoothed, which is physically sound; (ii) the shear-stress is correctly negative in correspondence with the recirculation. It can be noted that the position of the jump is better predicted by the 1-D shallow-water model with Watson's friction.

\subsubsection{Hydraulic jump on an inclined surface}

In this final section the effects of the inclination of the plate on the hydraulic jump are investigated. The topography is defined as $z=a x$ and $a$ varies between -1.6 and 0.8 for three different values of $S, 0.5,1$ and 2. The values of $a$ are chosen such that the position of the hydraulic jump is not too close to the inlet or the outlet.

Figure 10 shows the liquid depth and the skin friction for all the cases with negative values of $a$. In this case, increasing the inclination of the plate the position of the hydraulic jump moves downstream due to the presence of a favourable component of the acceleration of gravity. The maximum height of the surface decreases and the hydraulic jump becomes less abrupt, i.e. the rise of the liquid depth after the jump is less rapid, leading to a more "gentle" hydraulic jump. In all the cases the liquid depth tends towards an 


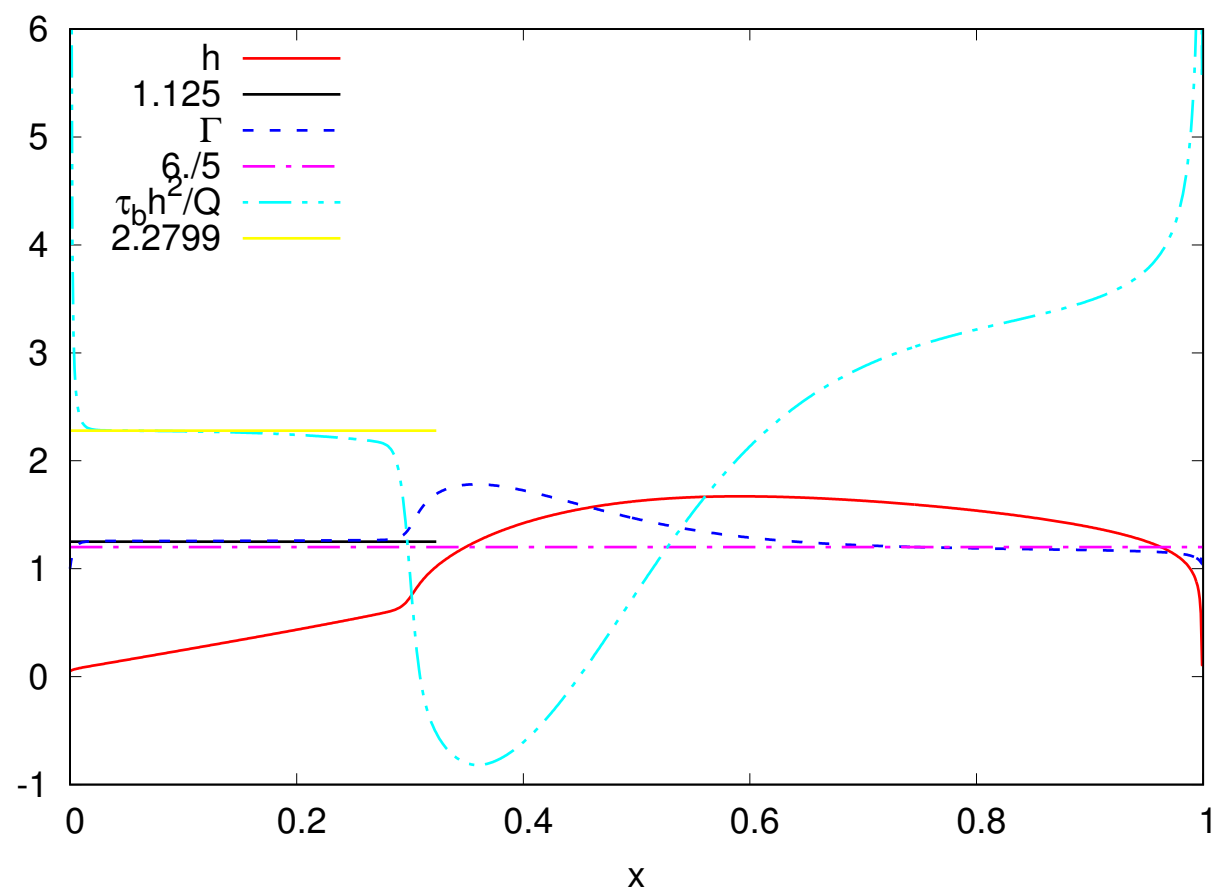

Figure 7: Plot of an example of resolution of system 28 for $\tilde{Q}=1$. Free surface position $\tilde{h}(\tilde{x})$ (solid red line), computed shape factor $\Gamma$ (dashed blue line) and computed skin friction $\partial \tilde{u} /\left.\partial \tilde{z}\right|_{0}$ (dash-dotted cyan line) rescaled by $\tilde{Q} / \tilde{h}^{2}$ as a function of $\tilde{x}$.

space to develop. The skin factor increases with the inclination of the plate and tends towards a constant value for the cases with $a=-1.6$. On the contrary, for positive values of $a$ the hydraulic jump moves towards the inlet, because gravity opposes to the flow. As the inclination increases the maximum height of the surface rises and the skin factor becomes larger. The results, in terms of water depth and skin factor, for the case with positive values of $a$ are drawn in figure 11 .

Figure 12 a shows the position of the jump $x_{j}$, computed as the location of the point with maximum second derivative of the liquid depth, as a function of $a$. With the hydraulic jump located closer to the inlet, for the case with horizontal plate, the position of the jump varies faster for positive values of $a$. In contrast, for negative values of the inclination, it seems to reach a plateau around $a=-1.6$.

As observed before, the maximum height decreases for negative values of the inclination of the plate. We have seen that $h$ also decreases as an effect of increasing $S$. For this reason we show in figure $12 \mathrm{~b}$ the maximum height, normalized with the maximum height for the case with no inclination. The maximum liquid depth decreases faster for higher values of $S$ because for $S>1$ gravity is more effective.

\section{Conclusions}

This work presents a reduced set of Navier-Stokes equations leading to a kind of Prandtl system of equations (RNSP equations), obtained through the asymptotic thin-layer expansion. These are the Prandtl equations with different boundary conditions (Schlichting [49]) and they have already been derived on more phenomenological grounds [24, 25, 26. These thin-layer incompressible equations assume hydrostatic pressure by construction. If they are integrated over the depth of fluid, they give the Saint-Venant equations (or Shallow Water equations). In these reduced Navier-Stokes equations no hypothesis is made about the velocity profile, which is a result of the computations, whereas in the Saint-Venant equations a closure hypothesis is necessary.

An efficient numerical scheme issued from a large recent literature is proposed to solve the RNSP system using a discretization in several layers (as a discretization of the viscous diffusive term). The same numerical 


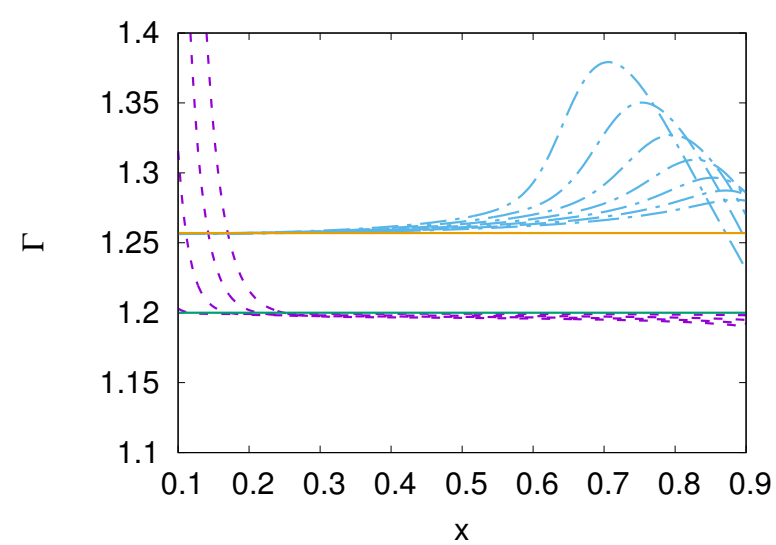

(a)

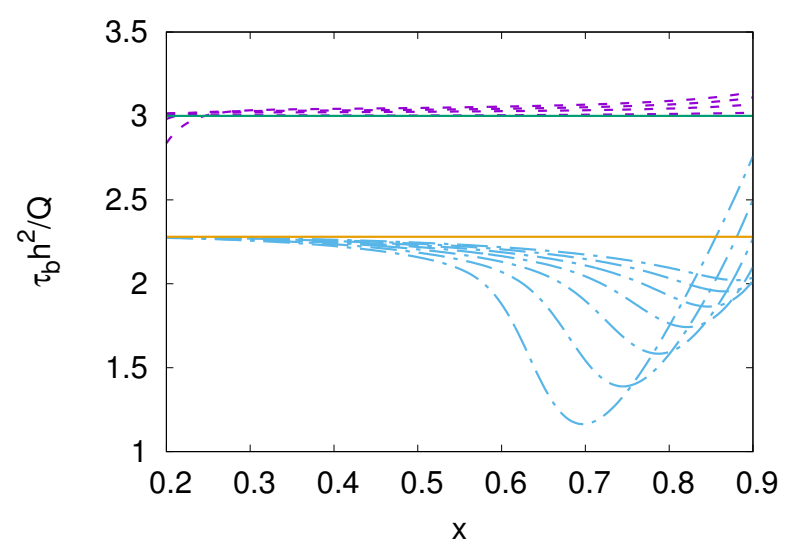

(b)

Figure 8: Plot of computed shape function $\Gamma=\frac{\tilde{h} \int \tilde{u}^{2} d \tilde{z}}{\left(\int \tilde{u} d \tilde{z}\right)^{2}}$ (Boussinesq coefficient) and reduced wall shear $\left.\frac{\partial \tilde{u}}{\partial \tilde{z}}\right|_{0} \frac{\tilde{h}^{2}}{\tilde{Q}}$ for $\tilde{Q}<.3$ (purple dashed lines) and $\tilde{Q}>1.3$ (cyan dashed-dot lines). Poiseuille is $6 . / 5$ and 3 (green solid lines), Watson is 1.25697 and 2.2799 (orange solid lines). Note that the value of $\Gamma$ is never 1 .

scheme solves also the standard Saint-Venant set of equations in one layer. We have thus clarified how the numerical boundary-layer approach to solve hydraulic jump problems and the numerical schemes developed to improve the solution of Saint-Venant equations are in fact intimately related.

For the sake of validation, the viscous collapse on a horizontal and on an inclined infinite flat plate is presented. In this case RNSP and Saint-Venant give the same self-similar solution. The next example is the hydraulic jump induced on a parietal jet over a finite plate. This case corresponds to a full balance of inertial, pressure and viscous terms. The steady solution of Higuera is recomputed. The hydraulic jump is no more a discontinuity (as in Saint-Venant) but is a region where the depth of the fluid increases continuously, on a short distance. A separation bubble appears under the jump, due to the strong deceleration. Compared with a one layer Saint-Venant model we showed the influence of the chosen profile (Watson or Poiseuille) on the wall friction. In the one layer Saint-Venant description friction at the wall is always positive whereas there is flow separation under the jump as computed first by Highera. Furthermore, we recomputed the Boussinesq velocity shape coefficient which is mainly approximated to unity in a one layer Saint-Venant model. We show that its value changes and remains of order one, but not equal to one, in the proposed examples.

To a certain extent, this is a beginning of response to Bélanger's question about the real shape of the hydraulic jump, see figure 13 . It is shown that the shape factor and friction are indeed different from the usual ones used in Shallow Water theory. Therefore, we have shown that the present Boundary Layer approach should be preferred when dealing with phenomena where several mechanisms occur concurrently, and for which the Saint-Venant approximation is too crude. Finally the plate is inclined and the change of position of the jump is discussed.

Concerning the perspectives, the extension in the transverse direction is straightforward and has been already integrated to the code. An open issue is related to the fact that the theoretical model discussed here does not take into account the possibility of dispersion, which may allow for solitary waves, "ondular bore" or "mascaret" [48.

An interesting development would be to solve the Navier-Stokes equations with this kind of method while being able to automatically and continuously switch between the hierarchy of models: Shallow Water / RNSP / Navier-Stokes, depending on the desired degree of accuracy. 




Figure 9: Plot of $\tilde{h}$ (lines) and (1/10) $\left.\frac{\partial \tilde{u}}{\partial \tilde{z}}\right|_{0}$ (symbols) for standard Saint Venant with a friction coefficient equa;l to Poiseuille's value 3 (purple); the same but with a friction coefficient equal to Watson's value 2.2799 (green), and finally Higuera's jump (blue) with multilayer resolution.

\section{Appendix}

All the codes are free to download on the Basilisk web pages:

http://basilisk.fr/

http://basilisk.fr/src/saint-venant.h

http://basilisk.fr/src/test/wind-driven.c

http://basilisk.fr/sandbox/M1EMN/Exemples/viscous_collapse.c

http://basilisk.fr/sandbox/M1EMN/Exemples/viscous_collapse_noSV.c

http://basilisk.fr/sandbox/M1EMN/Exemples/viscous_collapse_ML.c

http://basilisk.fr/sandbox/M1EMN/Exemples/viscolsqrt.c

http://basilisk.fr/sandbox/M1EMN/Exemples/viscous_collapsesqrt_ML.c

http://basilisk.fr/sandbox/M1EMN/Exemples/svdbvismult_hydrojump.c

http://basilisk.fr/src/test/higuera.c

http://basilisk.fr/src/test/layered.c

\section{References}

[1] A. Saint-Venant, Théorie du mouvement non permanent des eaux avec applications aux crues des rivières et à l'introduction des marées dans leur lit, C. R. Acad. Sci. Paris 73 (1871) 147-154.

[2] H. Chanson, Hydraulics of open channel flow, Butterworth-Heinemann, 2004.

[3] N. J. Balmforth, R. V. Craster, A. C. Rust, R. Sassi, Viscoplastic flow over an inclined surface, Journal of non-newtonian fluid mechanics 139 (1-2) (2006) 103-127.

[4] E. F. Toro, Riemann solvers and numerical methods for fluid dynamics: a practical introduction, Springer Science \& Business Media, 2013

[5] J. Lighthill, M. Lighthill, Waves in fluids, Cambridge university press, 2001.

[6] J.-F. Gerbeau, B. Perthame, Derivation of viscous saint-venant system for laminar shallow water; numerical validation, Ph.D. thesis, INRIA (2000).

[7] A. Decoene, L. Bonaventura, E. Miglio, F. Saleri, Asymptotic derivation of the section-averaged shallow water equations for natural river hydraulics, Mathematical Models and Methods in Applied Sciences 19 (03) (2009) $387-417$. 


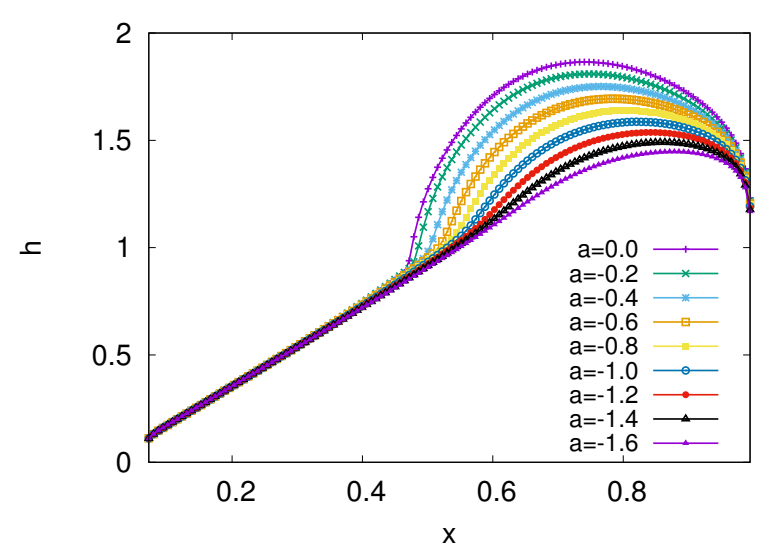

(a)

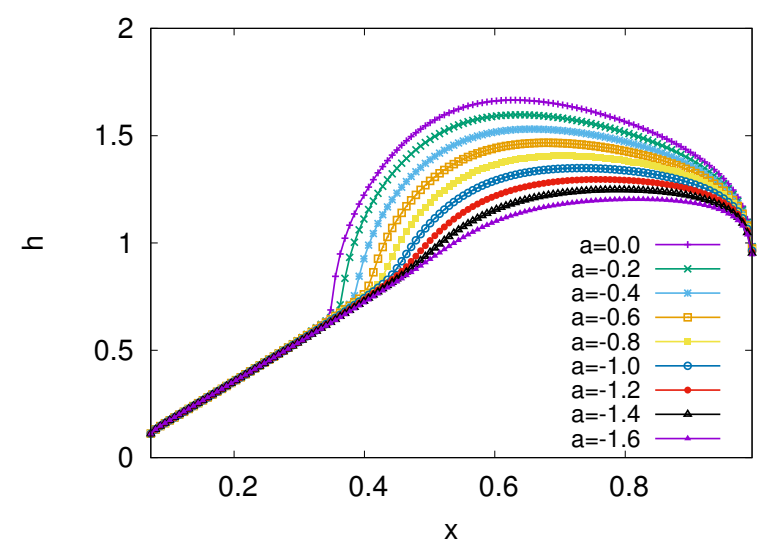

(c)



(e)

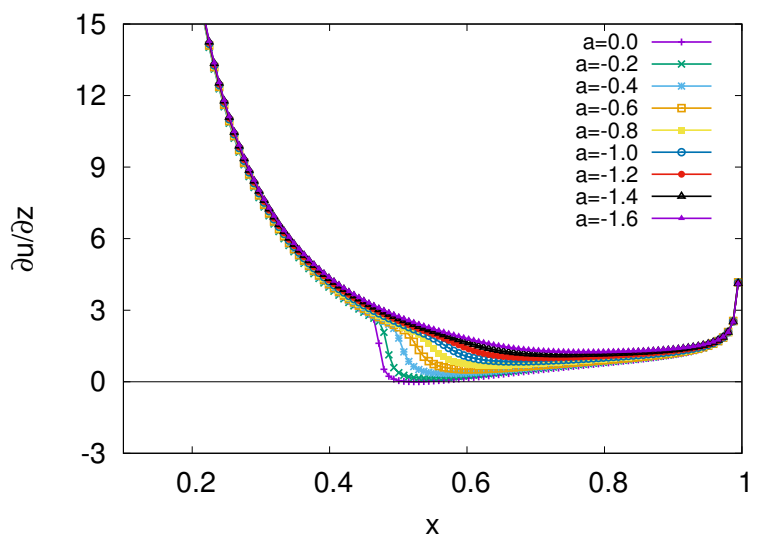

(b)

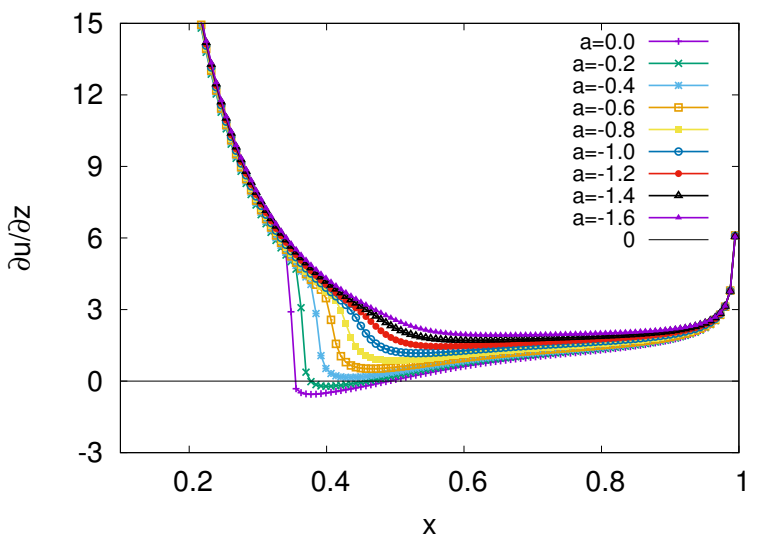

(d)

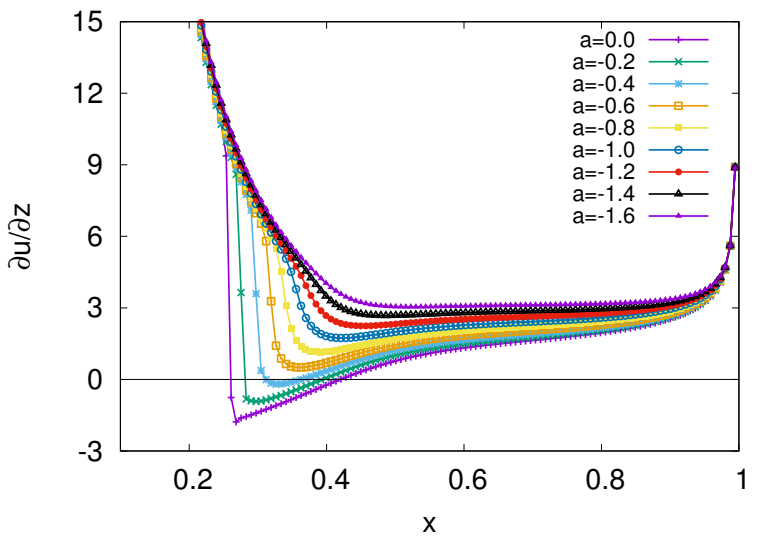

(f)

Figure 10: Water depth (a, c, e) and skin friction (b, d, f) for the case with negative value of $a$ at different value of $S$ (the Froude number is $\left.S^{-1 / 2}\right): S=0.5(\mathrm{a}, \mathrm{b}), S=1(\mathrm{c}, \mathrm{d}), S=2(\mathrm{e}, \mathrm{f})$. 


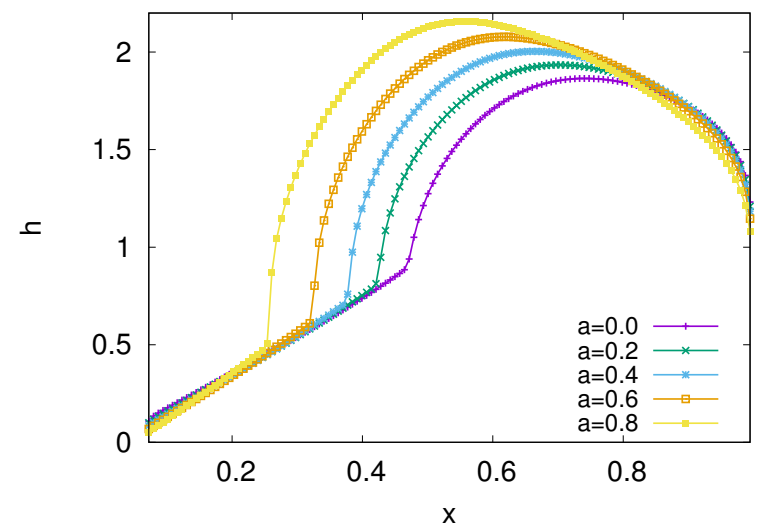

(a)

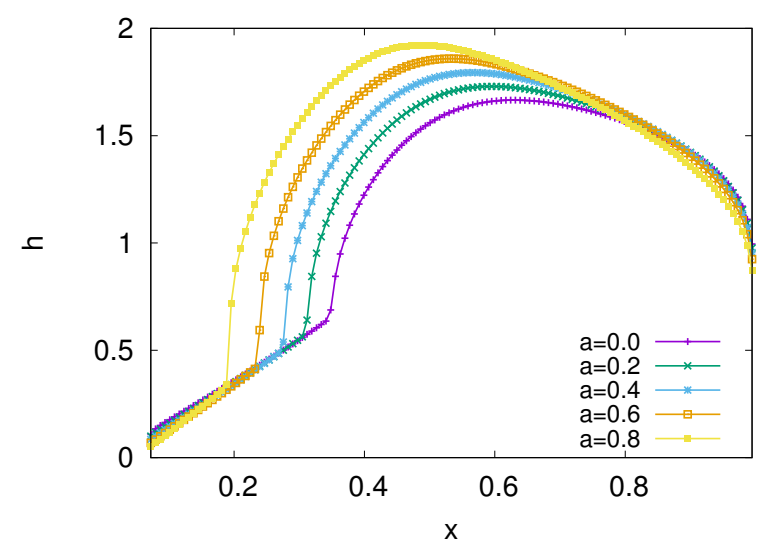

(c)



(e)

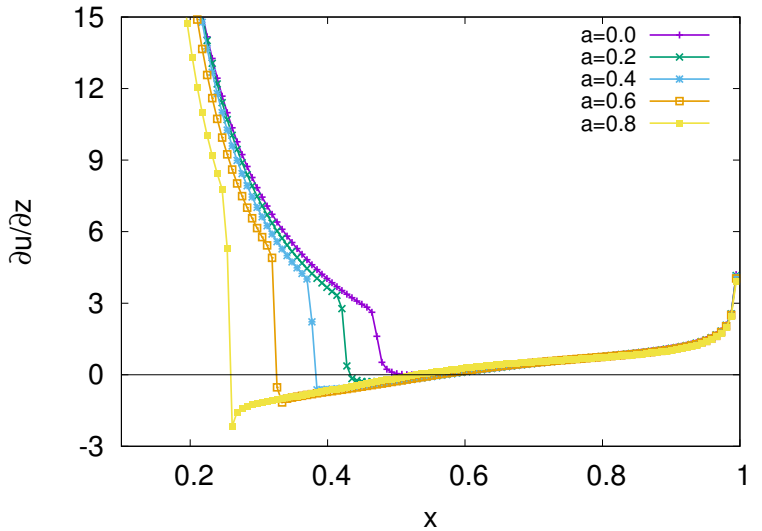

(b)



(d)

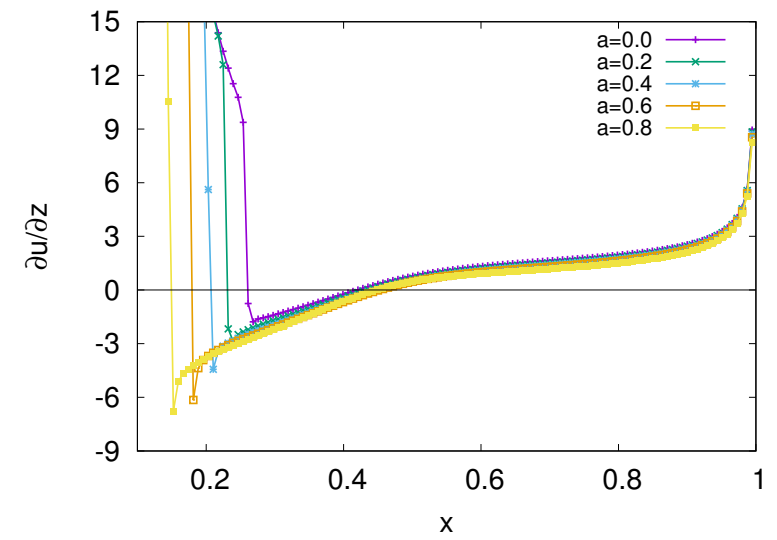

(f)

Figure 11: Water depth (a, c, e) and skin friction (b, d, f) for the case with positive value of $a$ at different value of $S: S=0.5$ $(\mathrm{a}, \mathrm{b}), S=1(\mathrm{c}, \mathrm{d}), S=2(\mathrm{e}, \mathrm{f})$. 




(a)



(b)

Figure 12: Position of the jump $x_{j}$ (a) and maximum height $h_{\max }$ (normalized by the maximum height for the case with horizontal surface $h_{0}$ ) (b) as function of the inclination $a$.

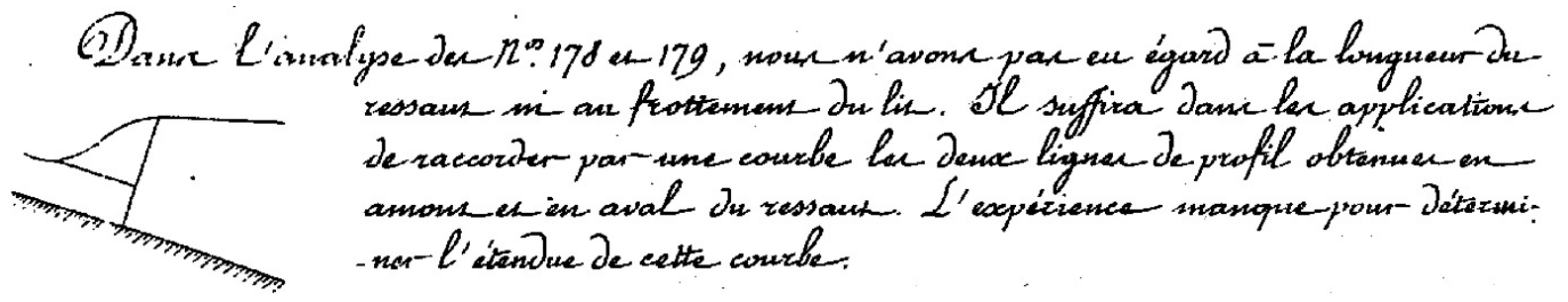

Figure 13: The sketch of the hydraulic jump by Bélanger 58 and the sentences about the influence of skin friction on the two levels of the jump: For the analysis of $n^{\circ} 178$ and 179, we did not take into account either the length of the jump or the bed friction. It will be enough in applications to connect by a curve the two lines of profile obtained upstream and downstream of the jump. Experiments are lacking to determine the extent of this curve. 
[8] O. Delestre, Simulation du ruissellement d'eau de pluie sur des surfaces agricoles, Ph.D. thesis, Université d'Orléans (2010).

[9] F. Yang, D. Liang, Y. Xiao, Influence of boussinesq coefficient on depth-averaged modelling of rapid flows, Journal of Hydrology 559 (2018) 909 - 919. doi:https://doi.org/10.1016/j.jhydrol.2018.01.053 URL http://www.sciencedirect.com/science/article/pii/S0022169418300623

[10] S. Mejean, T. Faug, I. Einav, A general relation for standing normal jumps in both hydraulic and dry granular flows, Journal of Fluid Mechanics 816 (2017) 331-351. doi:10.1017/jfm.2017.82

[11] E. Audusse, A multilayer Saint-Venant system: Derivation and numerical validation, Discrete Contin. Dyn. Syst. Ser. B 5 (2005) 189-214.

[12] E. Audusse, M. O. Bristeau, A. Decoene, Numerical simulations of 3d free surface flows by a multilayer Saint-Venant model, Int. J. Numer. Methods Fluids 56 (2008) 331-350.

[13] E. Audusse, M. Bristeau, B. Perhame, J. Saint-Marie, A multilayer Saint-Venant System with mass exchanges for shallow water flows. Derivation and numerical validation, ESAIM: Mathematical Modelling and Numerical Analysis 45 (2011) $169-200$.

[14] E. Audusse, M.-O. Bristeau, M. Pelanti, J. Sainte-Marie, Approximation of the hydrostatic navier-stokes system for density stratified flows by a multilayer model: kinetic interpretation and numerical solution, Journal of Computational Physics 230 (9) (2011) 3453-3478.

[15] E. Audusse, F. Benkhaldoun, S. Sari, M. Seaid, P. Tassi, A fast finite volume solver for multi-layered shallow water flows with mass exchange, Journal of Computational Physics 272 (2014) $23-45$

[16] B. D. Martino, B. Haspot, Y. Penel, Global stability of weak solutions for a multilayer saint-venant model with interactions between layers, Nonlinear Analysis 163 (2017) 177 - 200.

[17] M.-O. Bristeau, C. Guichard, B. DI Martino, J. Sainte-Marie, Layer-averaged Euler and Navier-Stokes equations, Communications in Mathematical Sciencesdoi:10.4310/CMS.2017.v15.n5.a3

[18] Multilayer shallow water models with locally variable number of layers and semi-implicit time discretization, Journal of Computational Physics 364 (2018) $209-234$.

[19] V. Prokofev, Multilayer open flow model: A simple pressure correction method for wave problems, International Journal for Numerical Methods in Fluids 86 (8) (2018) 519-540.

[20] 2d granular flows with the $\mu$-(i) rheology and side walls friction: A well-balanced multilayer discretization, Journal of Computational Physics 356 (2018) $192-219$.

[21] C. Ruyer-Quil, P. Manneville, Modeling film flows down inclined planes, The European Physical Journal B - Condensed Matter and Complex Systems 6 (2) (1998) 277-292.

[22] C. Ruyer-Quil, P. Manneville, Improved modeling of flows down inclined planes, The European Physical Journal B Condensed Matter and Complex Systems 15 (2) (2000) 357-369.

[23] James, François, Lagrée, Pierre-Yves, Le, Minh H., Legrand, Mathilde, Towards a new friction model for shallow water equations through an interactive viscous layer ESAIM: M2AN 53 (1) (2019) 269-299. doi:10.1051/m2an/2018076 URL https ://doi .org/10.1051/m2an/2018076

[24] R. Bowles, F. Smith, The standing hydraulic jump: theory, computations and comparisons with experiments, Journal of Fluid Mechanics 242 (1992) 145-168.

[25] F. J. Higuera, The hydraulic jump in a viscous laminar flow, J. Fluid Mech. 274 (1994) 69-92.

[26] F. Higuera, The circular hydraulic jump, Physics of Fluids 9 (5) (1997) 1476-1478.

[27] H. Chanson, Development of the bélanger equation and backwater equation by jean-baptiste bélanger (1828), Journal of Hydraulic Engineering 135 (3) (2009) 159-163.

340 [28] E. Watson, The radial spread of a liquid jet over a horizontal plane, Journal of Fluid Mechanics 20 (3) (1964) $481-499$.

[29] S. Watanabe, V. Putkaradze, T. Bohr, Integral methods for shallow free-surface flows with separation, Journal of Fluid Mechanics 480 (2003) 233-265.

[30] A. Duchesne, L. Lebon, L. Limat, Constant froude number in a circular hydraulic jump and its implication on the jump radius selection, EPL (Europhysics Letters) 107 (5) (2014) 54002.

[31] N. Rojas, M. Argentina, E. Cerda, E. Tirapegui, Inertial lubrication theory, Physical review letters 104 (18) (2010) 187801.

[32] R. Dasgupta, R. Govindarajan, Nonsimilar solutions of the viscous shallow water equations governing weak hydraulic jumps, Physics of Fluids 22 (11) (2010) 112108.

[33] O. Thual, L. Lacaze, M. Mouzouri, B. Boutkhamouine, Critical slope for laminar transcritical shallow-water flows, Journal of Fluid Mechanics 783 .

350 [34] R. Dasgupta, G. Tomar, R. Govindarajan, Numerical study of laminar, standing hydraulic jumps in a planar geometry, The European Physical Journal E 38 (5) (2015) 45.

[35] E. D. Fernández-Nieto, E. Koné, T. C. Rebollo, A multilayer method for the hydrostatic navier-stokes equations: a particular weak solution, Journal of Scientific Computing 60 (2) (2014) 408-437.

[36] S. B. Savage, K. Hutter, The motion of a finite mass of granular material down a rough incline, Journal of fluid mechanics 199 (1989) 177-215.

[37] P.-Y. Lagrée, G. Saingier, S. Deboeuf, L. Staron, S. Popinet, Granular front for flow down a rough incline: about the value of the shape factor in depths averaged models, in: EPJ Web of Conferences, Vol. 140, EDP Sciences, 2017 , p. 03046.

[38] E. D. Fernández-Nieto, J. Garres-Díaz, A. Mangeney, G. Narbona-Reina, A multilayer shallow model for dry granular flows with the $\mu$ (i)-rheology: application to granular collapse on erodible beds, Journal of Fluid Mechanics 798 (2016) $643-681$.

[39] A. R. Ghigo, J.-M. Fullana, P.-Y. Lagrée, A 2d nonlinear multiring model for blood flow in large elastic arteries, Journal of Computational Physics 350 (2017) 136-165.

[40] P.-Y. Lagrée, Interactive boundary layer (ibl), in: Asymptotic methods in fluid mechanics: survey and recent advances, 
Springer, 2010, pp. 247-286.

[41] M. Werle, P. M. VIJGEN, W. Hankey, Initial conditions for the hypersonic-shock/boundary-layer interaction problem., AIAA Journal 11 (4) (1973) 525-530.

[42] W. Schneider, M. Wasel, Breakdown of the boundary-layer approximation for mixed convection above a horizontal plate, International journal of heat and mass transfer 28 (12) (1985) 2307-2313.

[43] H. Steinrück, Mixed convection over a cooled horizontal plate: non-uniqueness and numerical instabilities of the boundarylayer equations, Journal of Fluid Mechanics 278 (1994) 251-265.

[44] P.-Y. Lagrée, Removing the marching breakdown of the boundary-layer equations for mixed convection above a horizontal plate, International journal of heat and mass transfer 44 (17) (2001) 3359-3372.

[45] R. Bowles, F. Smith, The standing hydraulic jump: theory, computations and comparisons with experiments, Journal of Fluid Mechanics 242 (1992) 145-168.

[46] J. Gajjar, F. Smith, On hypersonic self-induced separation, hydraulic jumps and boundary layers with algebraic growth, Mathematika 30 (1) (1983) 77-93.

[47] http://basilisk.fr.

[48] S. Popinet, A quadtree-adaptive multigrid solver for the serre-green-naghdi equations, Journal of Computational Physics 302 (2015) 336-358.

380 [49] H. Schlichting, Boundary layer theory, Springer, 1987.

[50] H. Tennekes, J. L. Lumley, J. Lumley, et al., A first course in turbulence, MIT press, 1972.

[51] G. Kirstetter, J. Hu, O. Delestre, F. Darboux, P.-Y. Lagrée, S. Popinet, J.-M. Fullana, C. Josserand, Modeling rain-driven overland flow: Empirical versus analytical friction terms in the shallow water approximation, Journal of Hydrology 536 (2016) $1-9$.

[52] S. B. Pope, Turbulent Flows, Cambridge University Press, 2000. doi:10.1017/CB09780511840531

[53] A. Kurganov, D. Levy, Central-upwind schemes for the saint-venant system, Math. Modell. and Numer. Anal. 36 (2002) 397-425.

[54] E. Audusse, F. Bouchut, M. Bristeau, R. Klein, B. Perthame, A fast and stable well-balanced scheme with hydrostatic reconstruction for shallow water flows, SIAM J. Scien. Comp. (2004) 2050-2065.

[55] H. E. Huppert, The propagation of two-dimensional and axisymmetric viscous gravity currents over a rigid horizontal surface, Journal of Fluid Mechanics 121 (1982) 43-58.

[56] H. E. Huppert, Flow and instability of a viscous current down a slope, Nature 300 (5891) (1982) 427.

[57] N. Shankar, H. Cheong, S. Sankaranarayanan, Multilevel finite-difference model for three-dimensional hydrodynamic circulation, Ocean Engineering 24 (9) (1997) 785 - 816.

[58] J.-B. Belanger, Notes sur le cours d'hydraulique. session 1849-1850, bibliothèque numeŕique patrimoniale des ponts et chaussées

URL https://patrimoine.enpc.fr/document/ENPC02_COU_4_2382_1849 